\title{
BRS Cohomology of the Chiral Superfield
}

\author{
J. A. Dixon $\star$ \\ Theory Group, Physics Department, University of Texas, Austin, TX 78712, USA \\ Received November 9, 1989; in revised form November 15, 1990
}

\begin{abstract}
As a start in a search for possible undiscovered anomalies which might break supersymmetry, a general calculation of BRS cohomology for the Wess Zumino chiral multiplet is performed. The calculation is done using spectral sequences in Fock space. It encompasses the vector space of all integrated local polynomials in the fields and their derivatives. This calculation shows that the BRS cohomology space contains an infinite number of polynomials with ghost charge one. Examples of these polynomials are given. All presently known examples possess uncontracted Lorentz spinor (and possibly vector) indices. A simple extension of these results to super Yang Mills theory indicates that there may be previously unnoticed anomalies in that theory.
\end{abstract}

\section{Table of Contents}

I. Introduction . . . . . . . . . . . . . . . . . . . . . . . . 170

1. Discussion . . . . . . . . . . . . . . . . . . . . . . . . . . . 170

2. Action and Supersymmetry Invariance . . . . . . . . . . . . . . . . . 172

3. Remarks about Superspace . . . . . . . . . . . . . . . . . . . . . 173

4. Quick Review of Procedure . . . . . . . . . . . . . . . . . . . . . 174

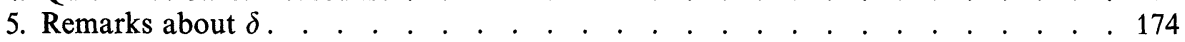

6. A Grading Operator and Its Spectral Sequence . . . . . . . . . . . . . . 175

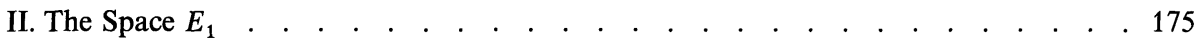

7. Calculation of $\Delta_{0}$ and Equations for $E_{1}$. . . . . . . . . . . . . . . . . . . 175

8. Ghost Degree, Form Degree, and Dimension. . . . . . . . . . . . . . . 176

9. Projection Operators $\Pi_{1}$ for the Space $E_{1}$. . . . . . . . . . . . . . . . . . . . . . . 179

9.1. A Useful Form of the Equations for $E_{1}$. . . . . . . . . . . . . . . . . . . . 179

9.2. Examination of the $\Lambda_{i}$ Operators . . . . . . . . . . . . . . . . . 179

9.3. The Projection Operator $\Pi_{A=0}$. $\quad . \quad$. . . . . . . . . . . . . . . . . . . . . . . . $\quad .180$

9.4. The Projection Operator $\Pi_{T=0}$. $\quad . \quad$. . . . . . . . . . . . . . . . . . . . . 182

* Research supported in part by the Robert A. Welch Foundation and NSF Grant PHY 9009850 
9.5. The Projection Operator $\Pi_{Q=0}$. . . . . . . . . . . . . . . . . . . . . . . . 182

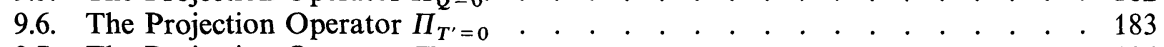

9.7. The Projection Operator $\Pi_{N_{1}=0}$. $\quad$. . . . . . . . . . . . . . . . . . . . . . . . . . . 184

9.8. The Projection Operator $\Pi_{N_{1}}=q$. $\quad$. $\quad$. . . . . . . . . . . . . . . . . . . . . . . . . . 184

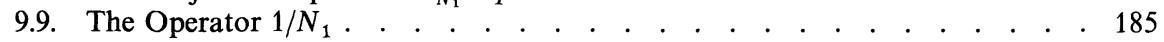

9.10. The Projection Operator for the Subspace where $\partial_{\mu} \partial_{\mu}^{\dagger}=0$. . . . . . . . . . 185

9.11. The Projection Operator $\Pi_{\varepsilon}$.

9.12. The Total Projection Operator $\Pi_{1}=\Pi_{2}$. $\quad$. . . . . . . . . . . . . . . . . 186

III. The Space $E_{3}=E_{\infty}$. . . . . . . . . . . . . . . . . . . . . . . . . . 186

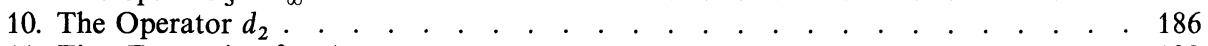

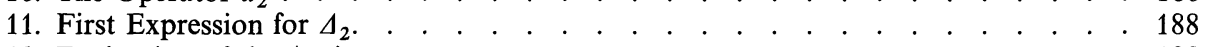

12. Evaluation of the Anticommutator . . . . . . . . . . . . . . . . . . 188

13. Second Expression for $\Delta_{2}$.

14. Second Form for the Anticommutator . . . . . . . . . . . . . . . . . . 189

15. A Simple Form for $\Delta_{2}$...$\quad$. . . . . . . . . . . . . . . . . . . . . . . . . . . . 190

16. The Operators $d_{r}$ are Zero for $r \geqq 3$. . . . . . . . . . . . . . . . . . . . . . . . . . 191

17. A Set of Equations for the Space $E_{3}=E_{\infty}$. . . . . . . . . . . . . . . . . . . . 191

18. Summary of the Equations for $E_{\infty}$. . . . . . . . . . . . . . . . . . . . . . . . 193

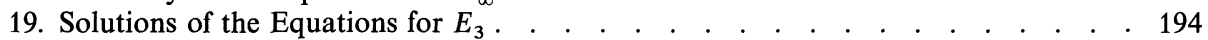

19.1 Superfields and the $\mathscr{G}=0$ Sector of $E_{\infty}$. . . . . . . . . . . . . . . . . . . 194

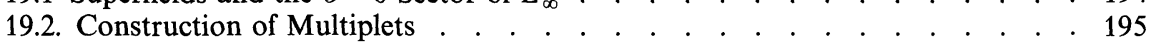

19.3. Some Polynomials with $\mathscr{G} \geqq 1$ : Solutions with no Spacetime Derivatives . . . 196

IV. Conclusion . . . . . . . . . . . . . . . . . . . . . . . . . . . 197

V. Appendices . . . . . . . . . . . . . . . . . . . . . . . . . . . 198

A. Summary of Basic Results and Notation . . . . . . . . . . . . . . . . 198

B. $\gamma$ Matrix Conventions and Formulae . . . . . . . . . . . . . . . . . . 199

\section{Part I. Introduction}

\section{Discussion}

Supersymmetry [6] is an appealing symmetry with some remarkable properties. It plays an important role in the only known way to reconcile quantum mechanics with gravitation - the superstring theory [5]. However little basis for comparison of supersymmetric theories with experiment has yet materialized. The basic reason for this is that it has not been found possible to understand the breaking of supersymmetry in a well motivated way. There is little uniqueness in the known possibilities.

The hope that motivates the present paper (and some others in preparation), is that there may exist supersymmetry anomalies that have not yet been uncovered and that these may be useful in unravelling some of the currently outstanding problems such as the origin of symmetry breaking and "dimensional reduction" in the superstring, and the origin of the huge mass splitting between weak and gravitational interactions. Of course it would not be surprising if the correct application of such anomalies turned out to be something quite different. At any rate it seems worthwhile to see whether any such anomalies exist, and to examine their properties if any are found.

It is well known that the invariants and anomalies in all gauge theories can be classified as elements of the cohomology space of the BRS operator acting in the space of local integrated polynomials made from the fields and their derivatives 
[1]. The anomalies are the elements in the cohomology space that have ghost charge $\mathscr{G}$ equals to one (or possibly higher [4]). The cohomology of the BRS operators in Yang-Mills theory and gravitation is known [2]. There are a finite number of polynomials in the $\mathscr{G}=1$ part of the cohomology space and most or all of them correspond to anomalies in the theory. The coefficients depend on the details of the theory. These anomalies are all of low canonical dimension.

It has generally been assumed that making a theory supersymmetric does not materially change the anomaly structure of the theory. It has been expected that anomalies in supersymmetric theories would be simply the old anomalies put into supersymmetric form somehow. This expectation is based on results which have been obtained for some specific theories and dimensions. For example the renormalization of the action for supersymmetric Yang-Mills theory in four dimensions has been shown to be afflicted by no new anomalies beyond the well known chiral ones in a supersymmetric form [10]. However it is not known what happens for other supersymmetric operators which have higher dimension. The situation for supersymmetric theories in more then four spacetime dimensions is even more unexplored.

Why take an interest in operators of dimension other than the dimension of the action? The simplest reason is that the problem is naturally solved using the present method in this general context. Another reason is that these operators occur in the operator product expansion and in the effective (non-renormalizable in appearance) field theories for gravity and supergravity. In turn these are the effective field theories in the zero slope limit of string and superstring theories [5].

Hence this gives a way to anaylze the superstring for supersymmetry anomalies. Recall that the gravitational anomalies for the superstring were first found by analyzing the effective supergravity theory limit. Such an approach is useful because we don't understand either Lorentz invariance or the action for superstring field theory very well. The present paper is intended to be the first in a series which will examine a number of these theories from the point of view of BRS cohomology.

The only known way to determine whether all the anomalies in these theories have been found is to compute the relevant cohomology space and rely on the BRS analysis to deduce that there are no anomalies if the cohomology space is empty. If the cohomology space is not empty, then one should calculate the Feynman diagrams that correspond with the objects in the cohomology space to see if there are anomalies in the theory. In other words the cohomology space tells one where to look for anomalies.

In [2], a method was described which permits the computation of the local BRS cohomology of any BRS operator. That paper was devoted to a complete description of a specific theory - the $S O(32)$ Yang-Mills theory in ten spacetime dimensions, which was chosen for analysis so that all the details could be described in a straightforward way. The description of [2] was complete in the sense that all local polynomials in the fields and their derivatives of all orders were included in the space that was analyzed.

This paper is devoted to a detailed examination of the local BRS cohomology of the Wess Zumino chiral multiplet [11] using the Fock space and spectral sequence methods of [2]. It is quite difficult to analyze the cohomology space of a field theory in this general way. That is why the simplest example of a supersymmetric theory has been chosen for this first attempt. It turns out that theories of the Wess Zumino type in less than four dimensions are rather trivial for present purposes. 
This can be established easily for simple models using the techniques in this paper. The basic reason for the difference seems to be that 4 dimensions admit complex chiral spinors, whereas lower dimensions do not. Of course we are not talking about the string theories here. However the four dimensional Wess Zumino model is certainly far from trivial.

\section{Action and Supersymmetry Invariance}

The (free quadratic) action for the Wess Zumino chiral model is:

$$
S=-\frac{1}{2} \int d^{4} x\left[\partial_{\mu} A \partial^{\mu} A+\partial_{\mu} B \partial^{\mu} B+\bar{\psi} \not \psi \psi-F^{2}-G^{2}\right],
$$

where $A$ and $F$ are scalar fields, $B$ and $G$ are pseudoscalar fields and $\psi$ is a 4-component real anticommuting (Majorana) spinor field. Conventions and some useful formulae can be found in Appendix B.

This action is invariant under the following supersymmetry and translational transformations:

$$
\begin{gathered}
\delta A=\bar{c} \psi+\varepsilon^{\mu} \partial_{\mu} A, \\
\delta B=\bar{c} \gamma_{5} \psi+\varepsilon^{\mu} \partial_{\mu} B, \\
\delta \psi=\not \partial\left[A+B \gamma_{5}\right] c+\left[F+G \gamma_{5}\right] c+\varepsilon^{\mu} \delta_{\mu} \psi, \\
\delta F=\bar{c} \not \phi \psi+\varepsilon^{\mu} \partial_{\mu} F, \\
\delta G=\bar{c} \gamma_{5} \not \psi+\varepsilon^{\mu} \partial_{\mu} G .
\end{gathered}
$$

Here $c$ is a constant (spacetime independent) commuting Majorana spinor and $\varepsilon_{\mu}$ is a constant anticommuting Lorentz vector. If we add the variations:

$$
\begin{gathered}
\delta \varepsilon_{\mu}=-\bar{c} \gamma_{\mu} c, \\
\delta c=0,
\end{gathered}
$$

then the variations acting on any field (counting $\varepsilon_{\mu}$ and $c$ as constant fields) satisfy the relation:

$$
\delta^{2}=0 .
$$

It will be assumed in the following that the reader is familiar with the methods and results of [2]. See Appendix A for a review of basic results and notation. Recall that there are two spaces of local polynomials: the integrated local polynomials $L$ and the unintegrated local polynomials $P$, and that these are related by the isomorphism between $L$ and the factor space of $P \bmod$ total derivatives:

$$
L \approx P / \partial P .
$$

Now clearly the operator $\delta$ given by:

$$
\begin{aligned}
\delta= & \int d^{4} x\left\{\bar{c} \psi \frac{\delta}{\delta A}+\bar{c} \gamma_{5} \psi \frac{\delta}{\delta B}+\left[\phi\left[A+B \gamma_{5}\right] c+\left[F+G \gamma_{5}\right] c\right] \frac{\delta}{\delta \psi}\right. \\
& \left.+\bar{c} \phi \psi \frac{\delta}{\delta F}+\bar{c} \gamma_{5} \not \psi \frac{\delta}{\delta G}\right\}
\end{aligned}
$$


is nilpotent when acting in the space $L$, because its square is proportional to the derivative operator and the integral of a total derivative in $L$ is taken to be zero.

The corresponding operator in the space $P$ is:

$$
\begin{aligned}
\delta= & \int d^{4} x\left\{\left(\bar{c} \psi+\varepsilon^{\mu} \partial_{\mu} A\right) \frac{\delta}{\delta A}+\left(\bar{c} \gamma_{5} \psi+\varepsilon^{\mu} \partial_{\mu} B\right) \frac{\delta}{\delta B}\right. \\
& +\left(\phi\left[A+B \gamma_{5}\right] c+\left[F+G \gamma_{5}\right] c+\varepsilon^{\mu} \partial_{\mu} \psi\right) \frac{\delta}{\delta \psi} \\
& \left.+\left(\bar{c} \phi \psi+\varepsilon^{\mu} \partial_{\mu} F\right) \frac{\delta}{\delta F}+\left(\bar{c} \gamma_{5} \phi \psi+\varepsilon^{\mu} \partial_{\mu} G\right) \frac{\delta}{\delta G}\right\}-\bar{c} \gamma_{\mu} c \varepsilon_{\mu}^{\dagger},
\end{aligned}
$$

and the cohomology space $H_{L}$ of (11) in $L$ is isomorphic to the cohomology space $H_{P}$ of (12) in $P$. The proof is exactly like the proof given in [2]. The remainder of this paper is devoted to an analysis of the cohomology of these operators in their spaces and to the isomorphism. As was done before, we analyze the cohomology of (11) by solving that of (12).

\section{Remarks about Superspace}

It might be thought that the superspace techniques that have been so successful in contructing and classifying the invariants and performing Feynman integrals for supersymmetric theories would be useful to the present problem. This is no doubt true. However I have not been able to use them yet in the actual solution of the cohomology problem. More accurately, an attempt to use superspace with the present method just results in the results recorded here. Taking derivatives with respect to an anticommuting Majorana spinor $\theta_{i}$ is just equivalent to using the various fields $A, B, \psi, F, G$ from the beginning.

Another problem with using superspace techniques here is that they depend on an anticommuting Majorana spinor $\varepsilon_{i}$ and we are concerned with a commuting Majorana spinor $c_{i}$. The useful feature of the anticommuting spinors is that products of a sufficient number of them eventually are zero. This is of course not true for a commuting spinor. The possibility of solving the problem in a simple way using superspace is a very real one, but it seems easier to use a more pedestrian approach for this initial attempt.

It will be clear however that once one knows something about the solutions of the cohomology problem, then superspace techniques are a natural way to construct those solutions.

In many ways it would be better to use a chiral complex notation instead of the present real notation. The results turn out to be chiral in nature. However, the present method is certainly equivalent and may well be the only way to solve some problems, such as $D=10$ super Yang-Mills.

A superspace method of doing BRS cohomology can be found in [9]. This paper has sometimes been interpreted to mean that the local BRS cohomology of supersymmetric theories must be trivial. However this interpretation is not correct for two reasons. One is that the methods of [9] are not applicable to chiral fields since the integral over $d^{4} \theta$ of a chiral quantity vanishes even though the chiral quantity itself does not vanish. The other is that no treatment of combined gauge and supersymmetry BRS invariance is attempted in [9], and the BRS cohomology 
of a combined operator is related to the BRS cohomology of one of its components in a very complex way - this is what gives rise to the spectral sequence.

\section{Quick Review of Procedure}

The techniques used in this paper were introduced in [2]. Here we shall find that the spectral sequence involves only a few steps. The steps necessary in the present case are:

- Choosing a grading that generates a useful spectral sequence.

- Calculating $\Delta_{0}$ and putting it in a useful form for the calculation of its Kernel.

- Constructing the projection operator $\Pi_{1}$ onto the space $E_{1}$ which is the Kernel of $\Delta_{0}$.

- Observing that $E_{1}=E_{2}$ because $\delta_{1}=0$.

- Finding suitable relations involving $d_{2}$ that make the calculation of $\Delta_{2}$ reasonably easy.

- Finding a form for $\Delta_{2}$ which makes the form of its kernel fairly transparent.

- Observing that $d_{r}=0$ for $r \geqq 3$ which implies that

$$
E_{3}=\left(\operatorname{Ker} \Delta_{2}\right) \cap E_{2}=E_{\infty} \approx H,
$$

where $H$ is the cohomology space that we want.

- Describing the polynomials in $E_{\infty}$ and the corresponding integrated polynomials in the isomorphic space $H$.

\section{Remarks about $\delta$}

As was remarked in [2], it is easiest to analyze the cohomology of the operator in $P$. That is our object in this paper. First let us introduce some notation:

$$
\delta=c_{i}\left[\Lambda_{i}+\nabla_{i}\right]+\varepsilon^{\mu} \partial_{\mu}-\bar{c} \gamma_{\mu} c \varepsilon_{\mu}^{\dagger},
$$

where:

$$
\Lambda_{i}=\int d^{4} x\left\{\left(\gamma_{0}\right)_{i j} \psi_{j} \frac{\delta}{\delta A}+\left(\gamma_{0} \gamma_{5}\right)_{i j} \psi_{j} \frac{\delta}{\delta B}+\left(F \delta_{j i}+G\left(\gamma_{5}\right)_{j i}\right) \frac{\delta}{\delta \psi_{j}}\right\}
$$

and:

$$
\begin{aligned}
\nabla_{i}= & \int d^{4} x\left\{\left[\left(\gamma^{\mu}\right)_{j i} \partial_{\mu} A+\left(\gamma^{\mu} \gamma_{5}\right)_{j i} \partial_{\mu} B\right] \frac{\delta}{\delta \psi_{j}}\right. \\
& \left.+\left(\gamma_{0} \gamma^{\mu}\right)_{i j} \partial_{\mu} \psi_{j} \frac{\delta}{\delta F}+\left(\gamma_{0} \gamma_{5} \gamma^{\mu}\right)_{i j} \partial_{\mu} \psi_{j} \frac{\delta}{\delta G}\right\} .
\end{aligned}
$$

The most obvious thing to try at the beginning is to simply calculate the operator:

$$
\Delta=\left[\delta+\delta^{\dagger}\right]^{2}
$$

and try to find its kernel.

This is the required cohomology space of $\delta$. However the resulting form of $\Delta$ is complicated, and it is not easy to find a form for it that consists of a sum of simple 
operators $O_{i}$ of the form:

$$
\Delta=\sum O_{i} O_{i}^{\dagger}
$$

which would result in the equations:

$$
O_{i}^{\dagger} H=0 .
$$

Since we do not find such a form, it is very difficult to characterize the solutions of $\Delta H=0$. The same problem occurred in [2] of course, and this calls for the use of the spectral sequence.

\section{A Grading Operator and its Spectral Sequence}

A grading $N$ that yields a useful spectral sequence is the following:

$$
N=c_{i} c_{i}^{\dagger}+\hat{N},
$$

where we define (for future purposes):

$$
\hat{N}=\int d^{4} x\left[3 A \frac{\delta}{\delta A}+3 B \frac{\delta}{\delta B}+2 \psi_{i} \frac{\delta}{\delta \psi_{i}}+F \frac{\delta}{\delta F}+G \frac{\delta}{\delta G}\right] .
$$

Sometimes we will use the notation:

$$
N(A)=\int d^{4} x\left[A \frac{\delta}{\delta A}\right] .
$$

The operator $\delta$ now splits up as follows:

$$
\delta=\delta_{0}+\delta_{2},
$$

where the operators $\delta_{i}$ satisfy the equations:

$$
\left[N, \delta_{i}\right]=i \delta_{i} .
$$

The detailed form of these operators is as follows:

$$
\begin{gathered}
\delta_{0}=c_{i} \Lambda_{i}+\varepsilon^{\mu} \partial_{\mu}, \\
\delta_{2}=c_{i} \nabla_{i}-\bar{c} \gamma_{\mu} c \varepsilon_{\mu}^{\dagger},
\end{gathered}
$$

where the operators $\Lambda$ and $\nabla$ are defined above. The feature that makes this $N$ a useful grading is that $\Lambda_{i}$ contains no derivatives. This makes a calculation of $\Delta_{0}$ a reasonably easy task, which will be accomplished in the next section.

\section{Part II. The Space $E_{1}$}

\section{Calculation of $\Delta_{0}$ and Equations for $E_{1}$}

The operator $\delta_{0}$ is our first concern, and we need to calculate

$$
\Delta_{0}=\delta_{0} \delta_{0}^{\dagger}+\delta_{0}^{\dagger} \delta_{0}
$$


from it as usual. A straightforward calculation of this operator using the usual notation of [2] yields the result:

$$
\begin{aligned}
& \Delta_{0}=\sum_{k=0}^{\infty} \frac{1}{k !}, \\
& \left(\left[c_{i} A_{\mu_{1} \mu_{2} \ldots \mu_{k}}-\left(\gamma_{5}\right)_{i j} c_{j} B_{\mu_{1} \mu_{2} \ldots \mu_{k}}\right]\left[c_{i} A_{\mu_{1} \mu_{2} \ldots \mu_{k}}-\left(\gamma_{5}\right)_{i l} c_{l} B_{\mu_{1} \mu_{2} \ldots \mu_{k}}\right]^{\dagger}\right. \\
& +\frac{1}{2}\left[\bar{\psi}_{\mu_{1} \mu_{2} \ldots \mu_{k}} c\right]\left[\bar{\psi}_{\mu_{1} \mu_{2} \ldots \mu_{k}} c\right]^{\dagger}+\frac{1}{2}\left[\bar{\psi}_{\mu_{1} \mu_{2} \ldots \mu_{k}} \gamma_{5} c\right]\left[\bar{\psi}_{\mu_{1} \mu_{2} \ldots \mu_{k}} \gamma_{5} c\right]^{\dagger} \\
& +\frac{1}{4}\left[\bar{\psi}_{\mu_{1} \mu_{2} \ldots \mu_{k}} \gamma_{\mu \nu} c\right]\left[\bar{\psi}_{\mu_{1} \mu_{2} \ldots \mu_{k}} \gamma_{\mu \nu} c\right]^{\dagger} \\
& \left.+\left[c_{i} F_{\mu_{1} \mu_{2} \ldots \mu_{k}}+\left(\gamma_{5}\right)_{i j} c_{j} G_{\mu_{1} \mu_{2} \ldots \mu_{k}}\right]\left[c_{i} F_{\mu_{1} \mu_{2} \ldots \mu_{k}}+\left(\gamma_{5}\right)_{i l} c_{l} G_{\mu_{1} \mu_{2} \ldots \mu_{k}}\right]^{\dagger}\right) \\
& +\Lambda_{i}^{\dagger} \Lambda_{i}+\varepsilon_{\mu}^{\dagger} \varepsilon_{\mu} N_{1}+\partial_{\mu} \partial_{\mu}^{\dagger} .
\end{aligned}
$$

This result can be obtained by noting that:

$$
\Delta_{0}=c_{i} c_{j}^{\dagger}\left\{\Lambda_{i}, \Lambda_{j}^{\dagger}\right\}+\Lambda_{j}^{\dagger} \Lambda_{j}+\varepsilon^{\nu \dagger} \varepsilon^{\mu}\left[\partial_{v}^{\dagger}, \partial_{\mu}\right]+\partial_{\mu} \partial_{\mu}^{\dagger},
$$

and then evaluating the anticommutator using the Fierz transformations to rearrange the spinorial parts so that they are manifestly a sum of non-negative operators. An operator analogous to $N_{1}$ appeared in [2]. $N_{1}$ is defined by:

$$
N_{1}=\int d^{4} x\left[A \frac{\delta}{\delta A}+B \frac{\delta}{\delta B}+\psi \frac{\delta}{\delta \psi}+F \frac{\delta}{\delta F}+G \frac{\delta}{\delta G}\right] \text {. }
$$

Since the above expression for $\Delta_{0}$ is a sum of non-negative operators, the subspace $E_{1}=\operatorname{Ker} \Delta_{0}$ satisfies the following equations:

$$
\begin{gathered}
{\left[c_{i} A_{\mu_{1} \mu_{2} \ldots \mu_{k}}-\left(\gamma_{5}\right)_{i l} c_{l} B_{\mu_{1} \mu_{2} \ldots \mu_{k}}\right]^{\dagger} E_{1}=0,} \\
{\left[\bar{\psi}_{\mu_{1} \mu_{2} \ldots \mu_{k}} c\right]^{\dagger} E_{1}=0,} \\
{\left[\bar{\psi}_{\mu_{1} \mu_{2} \ldots \mu_{k}} \gamma_{5} c\right]^{\dagger} E_{1}=0,} \\
{\left[\bar{\psi}_{\mu_{1} \mu_{2} \ldots \mu_{k}} \gamma_{\mu \nu} c\right]^{\dagger} E_{1}=0,} \\
{\left[c_{i} F_{\mu_{1} \mu_{2} \ldots \mu_{k}}+\left(\gamma_{5}\right)_{i l} c_{l} G_{\mu_{1} \mu_{2} \ldots \mu_{k}}\right]^{\dagger} E_{1}=0,} \\
\Lambda_{i} E_{1}=0, \\
\varepsilon_{\mu} N_{1} E_{1}=0, \\
\partial_{\mu}^{\dagger} E_{1}=0 .
\end{gathered}
$$

Commuting (or anticommuting) the operators appearing in these equations does not generate any new equations, but commutation of the operators in Eqs. (33) and (34) does suggest a new form which is equivalent to Eqs. (30)-(32) (the equivalence can be demonstrated by contracting the free spinor indices $(i, j)$ below with the complete set of $\gamma$ matrices):

$$
\left[\bar{\psi}_{i \mu_{1} \mu_{2} \ldots \mu_{k}} c_{j}-\bar{\psi}_{p \mu_{1} \mu_{2} \ldots \mu_{k}}\left(\gamma_{5}\right)_{p i}\left(\gamma_{5}\right)_{j q} c_{q}\right]^{\dagger} E_{1}=0 \text {. }
$$

\section{Ghost Degree, Form Degree, and Dimension}

In the corresponding calculation for Yang-Mills theory at this point it was possible to solve the equations for $E_{1}$ and for $E_{2}$ in a general way using simple algebra and the known results concerning the cohomology of the classical Lie algebras. It is not 
very easy to write down the general form of the solution of the equations for $E_{1}$ above. The problem is harder than the corresponding problem for $E_{1}$ or for $E_{2}$ in Yang-Mills theory.

Nevertheless the problem can be solved and the solution is presented below. Here we shall mention a few operators with nice properties that will be useful in classifying the solutions.

Note that the following set of equations generate all the equations for $E_{1}$ :

$$
\begin{gathered}
{\left[c_{i} F+\left(\gamma_{5}\right)_{i l} c_{l} G\right]^{\dagger} E_{1}=0,} \\
\Lambda_{i} E_{1}=0, \\
\varepsilon_{\mu} N_{1} E_{1}=0, \\
\partial_{\mu}^{\dagger} E_{1}=0,
\end{gathered}
$$

since the commutators such as:

$$
\left[\partial_{\mu}^{\dagger}, F_{\mu_{1} \mu_{2} \ldots \mu_{k}}^{\dagger}\right]=-F_{\mu_{1} \mu_{2} \ldots \mu_{k} \mu}^{\dagger}
$$

generate all the fields with more derivatives, and the commutator of $\Lambda_{i}$ and the operator in (33) generates Eq. (37) which is equivalent to Eqs. (30)-(32); and then the anticommutator of $\Lambda_{i}$ and the operator in Eq. (37) generates Eq. (29).

The process of searching for the general form of the subspace $E_{1}$ can be made systematic to some degree by noting the existence of the following operators and commutation relations:

$$
\begin{aligned}
& {\left[N_{1}, \delta\right]=0,} \\
& {[D, \delta]=0,} \\
& {[\mathscr{G}, \delta]=\delta,} \\
& {[\mathscr{F}, \delta]=\delta .}
\end{aligned}
$$

Here $N_{1}$ is the operator defined in Eq. (28), $D$ is the dimension operator with the following assignments of dimension: $c:-\frac{1}{2}, \varepsilon:-1, A: 1, B: 1, \psi: \frac{3}{2}, F: 2, G: 2, \partial: 1$, $x:-1 ; \mathscr{F}$ is the form degree operator, and $\mathscr{G}$ is the ghost degree (sometimes called ghost number or charge) operator:

$$
\begin{gathered}
\mathscr{F}=c_{i} c_{i}^{\dagger}+\varepsilon_{\mu} \varepsilon_{\mu}^{\dagger}, \\
\mathscr{G}=c_{i} c_{i}^{\dagger}+\varepsilon_{\mu} \varepsilon_{\mu}^{\dagger}-4=\mathscr{F}-4 .
\end{gathered}
$$

For the grading chosen below, all polynomials of interest will have $N(\varepsilon)=4$, so that in the spectral sequence that we use we will have $\mathscr{F}=N(c)+4$ and $\mathscr{G}=N(c)$. $D$ can be written in Fock space form as follows:

$$
D=-\frac{1}{2} c_{i} c_{i}^{\dagger}-\varepsilon_{\mu} \varepsilon_{\mu}^{\dagger}+D(f),
$$

where $D(f)$ is the contribution due to the fields and has the form:

$$
\begin{aligned}
D(f)= & \sum_{k=0}^{\infty} \frac{1}{k !}\left\{(k+1)\left[A_{\mu_{1} \mu_{2} \ldots \mu_{k}} A_{\mu_{1} \mu_{2} \ldots \mu_{k}}^{\dagger}+B_{\mu_{1} \mu_{2} \ldots \mu_{k}} B_{\mu_{1} \mu_{2} \ldots \mu_{k}}^{\dagger}\right]\right. \\
& +\left(k+\frac{3}{2}\right)\left[\psi_{i \mu_{1} \mu_{2} \ldots \mu_{k}} \psi_{i \mu_{1} \mu_{2} \ldots \mu_{k}}^{\dagger}\right] \\
& \left.+(k+2)\left[F_{\mu_{1} \mu_{2} \ldots \mu_{k}} F_{\mu_{1} \mu_{2} \ldots \mu_{k}}^{\dagger}+G_{\mu_{1} \mu_{2} \ldots \mu_{k}} G_{\mu_{1} \mu_{2} \ldots \mu_{k}}^{\dagger}\right]\right\} .
\end{aligned}
$$


The relations above give rise to the following relations for the operators $d_{r}$ of the spectral sequence:

$$
\begin{gathered}
{\left[N_{1}, d_{r}\right]=0,} \\
{\left[D, d_{r}\right]=0,} \\
{\left[\mathscr{G}, d_{r}\right]=d_{r},}
\end{gathered}
$$

and of course there is the relation

$$
\left[N, d_{r}\right]=r d_{r} .
$$

Hence one can look at the sectors of fixed $N_{1}$ and $D$ when examining the spaces $H$ and $E_{r}$ and the operators $d_{r}$. First we note that Eq. (40) is easily solved. Its solutions have the following form:

$$
E_{1}=P_{1}[A, B, \psi, F, G, \partial, c] T_{\varepsilon}^{4}+P_{1}[c, \varepsilon]
$$

where:

$$
T_{\varepsilon}^{4}=\varepsilon_{0} \varepsilon_{1} \varepsilon_{2} \varepsilon_{3}
$$

Here $P_{1}$ are polynomials dependent on the variables indicated and no others. The part $P_{1}[c, \varepsilon]$ which is independent of the spacetime dependent fields $A, B, \psi, F$, and $G$ is of no interest at present and its form will be ignored in this paper.

Another way of writing this result is:

$$
E_{1}=\left[\Pi_{N_{1}>0} \Pi_{\varepsilon=0}+\Pi_{N_{1}=0}\right] P[A, B, \psi, F, G, \partial, c, \varepsilon],
$$

where the projection operators $\Pi_{\varepsilon=0}$ and $\Pi_{N_{1}=0}$ are projection operators of the general form $\Pi_{X=0}$, which we define to satisfy the relations:

$$
X \Pi_{X=0}=0
$$

as well as the usual relations satisfied by an orthogonal projection operator:

$$
\Pi_{X=0}^{2}=\Pi_{X=0}^{\dagger}=\Pi_{X=0} .
$$

The projection operator $\Pi_{N_{1}>0}$ is of the general form $\Pi_{X>0}$. All operators $X$ which will appear in these expressions are hermitian and non-negative, so that this projection operator can be defined by:

$$
\Pi_{X=0}+\Pi_{X>0}=1 \text {, }
$$

where 1 is the identity operator in the Fock space.

An explicit form for these operators in terms of the Fock space operators will be given below.

Clearly the $\varepsilon$ dependence of the interesting part of $E_{1}$ is trivial and the problem now reduces to finding the general solution $P_{1}$ of the following equations:

$$
\begin{gathered}
{\left[c_{i} F+\left(\gamma_{5}\right)_{i l} c_{l} G\right]^{\dagger} P_{1}=0,} \\
\Lambda_{i} P_{1}=0, \\
\partial_{\mu}^{\dagger} P_{1}=0
\end{gathered}
$$




\section{Projection Operators $\Pi_{1}$ for the Space $E_{1}$}

\subsection{A Useful Form of the Equations for $E_{1}$}

There is a myriad of ways to write these equations and some ways are more useful for some purposes. The form given above is manifestly a sum of positive definite terms. The following form is useful for deducing the projection operators $\Pi_{1}$ that project onto the space $E_{1}$ :

$$
\begin{gathered}
\Lambda_{i} \Pi_{1}=0, \\
Q \Pi_{1}=0, \\
\partial_{\mu}^{\dagger} \Pi_{1}=0, \\
N_{1}\left[4-\varepsilon_{\mu} \varepsilon_{\mu}^{\dagger}\right] \Pi_{1}=0,
\end{gathered}
$$

where we define the operator $Q$ by:

$$
Q=\left\{N_{1} N_{1}^{\prime}-\tilde{N}_{1} \tilde{N}_{1}^{\prime}\right\} \Pi_{1} .
$$

Equation (65) above is equivalent to Eqs. (29)-(33) and can be obtained from them using rearrangement and Fierz transformations. The operators in 68 are defined below.

We already know the solution for (67). It turns out that we can construct the total operator $\Pi_{1}$ as a product and sum of simpler projection operators. The first task will be to find an operator to solve the $\Lambda$ equation, and this will be done in the next section.

\subsection{Examination of the $\Lambda_{i}$ Operators}

By writing out these operators in components, using the representation of the $\gamma$ matrices given in Appendix B, we find:

$$
\begin{aligned}
\Lambda_{1}= & \sum_{k=0}^{\infty} \frac{1}{k !}\left[-\psi_{4 \mu_{1} \mu_{2} \ldots \mu_{k}} A_{\mu_{1} \mu_{2} \ldots \mu_{k}}^{\dagger}+\psi_{2 \mu_{1} \mu_{2} \ldots \mu_{k}} B_{\mu_{1} \mu_{2} \ldots \mu_{k}}^{\dagger}\right. \\
& \left.+F_{\mu_{1} \mu_{2} \ldots \mu_{k}} \psi_{1 \mu_{1} \mu_{2} \ldots \mu_{k}}^{\dagger}+G_{\mu_{1} \mu_{2} \ldots \mu_{k}} \psi_{3 \mu_{1} \mu_{2} \ldots \mu_{k}}^{\dagger}\right], \\
\Lambda_{2}= & \sum_{k=0}^{\infty} \frac{1}{k !}\left[-\psi_{3 \mu_{1} \mu_{2} \ldots \mu_{k}} A_{\mu_{1} \mu_{2} \ldots \mu_{k}}^{\dagger}-\psi_{1 \mu_{1} \mu_{2} \ldots \mu_{k}} B_{\mu_{1} \mu_{2} \ldots \mu_{k}}^{\dagger}\right. \\
& \left.+F_{\mu_{1} \mu_{2} \ldots \mu_{k}} \psi_{2 \mu_{1} \mu_{2} \ldots \mu_{k}}^{\dagger}-G_{\mu_{1} \mu_{2} \ldots \mu_{k}} \psi_{4 \mu_{1} \mu_{2} \ldots \mu_{k}}^{\dagger}\right], \\
\Lambda_{3}= & \sum_{k=0}^{\infty} \frac{1}{k !}\left[\psi_{2 \mu_{1} \mu_{2} \ldots \mu_{k}} A_{\mu_{1} \mu_{2} \ldots \mu_{k}}^{\dagger}+\psi_{4 \psi_{1} \mu_{2} \ldots \mu_{k}} B_{\mu_{1} \mu_{2} \ldots \mu_{k}}^{\dagger}\right. \\
& \left.+F_{\mu_{1} \mu_{2} \ldots \mu_{k}} \psi_{3 \mu_{1} \mu_{2} \ldots \mu_{k}}^{\dagger}-G_{\mu_{1} \mu_{2} \ldots \mu_{k}} \psi_{1 \mu_{1} \mu_{2} \ldots \mu_{k}}^{\dagger}\right], \\
\Lambda_{4}= & \sum_{k=0}^{\infty} \frac{1}{k !}\left[\psi_{1 \mu_{1} \mu_{2} \ldots \mu_{k}} A_{\mu_{1} \mu_{2} \ldots \mu_{k}}^{\dagger}-\psi_{3 \mu_{1} \mu_{2} \ldots \mu_{k}} B_{\mu_{1} \mu_{2} \ldots \mu_{k}}^{\dagger}\right. \\
& \left.+F_{\mu_{1} \mu_{2} \ldots \mu_{k}} \psi_{4 \mu_{1} \mu_{2} \ldots \mu_{k}}^{\dagger}+G_{\mu_{1} \mu_{2} \ldots \mu_{k}} \psi_{2 \mu_{1} \mu_{2} \ldots \mu_{k}}^{\dagger}\right],
\end{aligned}
$$


where the first index $i$ on $\psi_{i \mu_{1} \mu_{2} \ldots \mu_{k}}$ is the spinor index in the column spinor:

$$
\psi_{i}=\left(\begin{array}{l}
\psi_{1} \\
\psi_{2} \\
\psi_{3} \\
\psi_{4}
\end{array}\right)
$$

These operators and their adjoints obey the following algebra:

$$
\begin{gathered}
\left\{\Lambda_{i}, \Lambda_{j}\right\}=0, \\
\left\{\Lambda_{i}^{\dagger}, \Lambda_{j}\right\}=\delta_{i j} N_{1}+\left(\gamma_{5}\right)_{i j} \tilde{N}_{1},
\end{gathered}
$$

where $N_{1}$ was defined in Eq. (28) and $\tilde{N}_{1}$ is defined as follows:

$$
\begin{aligned}
\tilde{N}_{1}= & \sum_{k=0}^{\infty} \frac{1}{k !}\left[A_{\mu_{1} \mu_{2} \ldots \mu_{k}} B_{\mu_{1} \mu_{2} \ldots \mu_{k}}^{\dagger}-B_{\mu_{1} \mu_{2} \ldots \mu_{k}} A_{\mu_{1} \mu_{2} \ldots \mu_{k}}^{\dagger}\right. \\
& +\psi_{3 \mu_{1} \mu_{2} \ldots \mu_{k}} \psi_{1 \mu_{1} \mu_{2} \ldots \mu_{k}}^{\dagger}-\psi_{1 \mu_{1} \mu_{2} \ldots \mu_{k}} \psi_{3 \mu_{1} \mu_{2} \ldots \mu_{k}}^{\dagger} \\
& +\psi_{2 \mu_{1} \mu_{2} \ldots \mu_{k}} \psi_{4 \mu_{1} \mu_{2} \ldots \mu_{k}}^{\dagger}-\psi_{4 \mu_{1} \mu_{2} \ldots \mu_{k}} \psi_{2 \mu_{1} \mu_{2} \ldots \mu_{k}}^{\dagger} \\
& \left.+G_{\mu_{1} \mu_{2} \ldots \mu_{k}} F_{\mu_{1} \mu_{2} \ldots \mu_{k}}^{\dagger}-F_{\mu_{1} \mu_{2} \ldots \mu_{k}} G_{\mu_{1} \mu_{2} \ldots \mu_{k}}^{\dagger}\right] \\
= & \sum_{k=0}^{\infty} \frac{1}{k !}\left[A_{\mu_{1} \mu_{2} \ldots \mu_{k}} B_{\mu_{1} \mu_{2} \ldots \mu_{k}}^{\dagger}-B_{\mu_{1} \mu_{2} \ldots \mu_{k}} A_{\mu_{1} \mu_{2} \ldots \mu_{k}}^{\dagger}\right. \\
& +\psi_{i \mu_{1} \mu_{2} \ldots \mu_{k}}\left(\gamma_{5}\right)_{i j} \psi_{j \mu_{1} \mu_{2} \ldots \mu_{k}}^{\dagger} \\
& \left.+G_{\mu_{1} \mu_{2} \ldots \mu_{k}} F_{\mu_{1} \mu_{2} \ldots \mu_{k}}^{\dagger}-F_{\mu_{1} \mu_{2} \ldots \mu_{k}} G_{\mu_{1} \mu_{2} \ldots \mu_{k}}^{\dagger}\right]
\end{aligned}
$$

and we note that:

$$
\begin{gathered}
\tilde{N}_{1}=-\tilde{N}_{1}^{\dagger} \\
N_{1}=N_{1}^{\dagger}
\end{gathered}
$$

and we also note that:

$$
\begin{aligned}
& {\left[\Lambda_{i}, \tilde{N}_{1}\right]=0,} \\
& {\left[\Lambda_{i}, N_{1}\right]=0,} \\
& {\left[N_{1}, \tilde{N}_{1}\right]=0 .}
\end{aligned}
$$

\subsection{The Projection Operator $\Pi_{\Lambda=0}$}

With the help of the foregoing, we can construct the orthogonal projection operator onto the subspace satisfying the equation:

$$
\Lambda_{i}^{\dagger} \Lambda_{i} \Pi_{\Lambda=0}=\Lambda_{i} \Pi_{\Lambda=0}=0 \text {. }
$$

It is

$$
\Pi_{\Lambda=0}=\Pi_{T=0} \Pi_{\Lambda=0,(T=0)}+\Pi_{T>0} \Pi_{\Lambda=0,(T>0)},
$$

where the non-negative hermitian operator $T$ is defined by:

$$
T=N_{1}^{2}+\tilde{N}_{1}^{2} \text {. }
$$


An illuminating way to see that $T$ is non-negative is to write the operators in the form:

$$
\begin{gathered}
N_{1}=f_{i}^{a} f_{i}^{a \dagger}, \\
\tilde{N}_{1}=\varepsilon_{i j} f_{i}^{a} f_{j}^{a \dagger} .
\end{gathered}
$$

Here $i, j=1,2$ (not to be confused with a spinor index) is an index that distinguishes the fields in each "pair"

$$
\left(A, B ; G, F ; \psi_{3}, \psi_{1} ; \psi_{2}, \psi_{4}\right)
$$

from each other and the index $a, b$ distinguishes the different pairs of fields from each other and also summarizes all the other indices $\left(\mu_{1} \ldots \mu_{k}\right)$. It is then an easy exercise to show that $T$ is non-negative using the simple identities:

$$
\varepsilon_{i j} \varepsilon_{k l}=\delta_{i k} \delta_{j l}-\delta_{i l} \delta_{j k} .
$$

The projection operator $\Pi_{\Lambda=0,(T>0)}$ has the form:

$$
\begin{aligned}
\Pi_{\Lambda=0,(T>0)}= & \frac{1}{T^{2}} \Lambda_{1} \Lambda_{2} \Lambda_{3} \Lambda_{4} \Lambda_{4}^{\dagger} \Lambda_{3}^{\dagger} \Lambda_{2}^{\dagger} \Lambda_{1}^{\dagger} \\
= & 1-\frac{N_{1}}{T}\left[\Lambda_{i}^{\dagger} \Lambda_{i}\right]-\frac{\tilde{N}_{1}}{T}\left[\Lambda_{i}^{\dagger}\left(\gamma_{5}\right)_{i j} \Lambda_{j}\right] \\
& +\frac{1}{T}\left[\Lambda_{i}^{\dagger} \Lambda_{j}^{\dagger} \Lambda_{j} \Lambda_{i}\right]+\ldots \\
& +\frac{1}{T^{2}} \Lambda_{1}^{\dagger} \Lambda_{2}^{\dagger} \Lambda_{3}^{\dagger} \Lambda_{4}^{\dagger} \Lambda_{4} \Lambda_{3} \Lambda_{2} \Lambda_{1},
\end{aligned}
$$

where the generalized inverse operator $\frac{1}{T}=\frac{1}{N_{1}^{2}+\tilde{N}_{1}^{2}}$ is defined to satisfy

$$
\left[N_{1}^{2}+\tilde{N}_{1}^{2}\right] \frac{1}{N_{1}^{2}+\tilde{N}_{1}^{2}}=\Pi_{T>0}=1-\Pi_{T=0} .
$$

We shall not need the explicit form of this operator in the following, and will just leave it in the form given.

The other projection operator is:

$$
\begin{aligned}
\Pi_{\Lambda=0,(T=0)} & =\frac{1}{2 N_{1}}\left[\Lambda_{1} \Lambda_{1}^{\dagger}+\Lambda_{3} \Lambda_{3}^{\dagger}\right] \frac{1}{2 N_{1}}\left[\Lambda_{2} \Lambda_{2}^{\dagger}+\Lambda_{4} \Lambda_{4}^{\dagger}\right] \\
& =\left\{1-\frac{1}{2 N_{1}}\left[\Lambda_{1}^{\dagger} \Lambda_{1}+\Lambda_{3}^{\dagger} \Lambda_{3}\right]\right\}\left\{1-\frac{1}{2 N_{1}}\left[\Lambda_{2}^{\dagger} \Lambda_{2}+\Lambda_{4}^{\dagger} \Lambda_{4}\right]\right\} \\
& =\left\{1-\frac{1}{2 N_{1}}\left[\Lambda_{i}^{\dagger} \Lambda_{i}\right]+\ldots\right\} .
\end{aligned}
$$

The demonstration that this latter operator satisfies the necessary equations uses the fact that:

$$
L_{1,3}=\Lambda_{1} \Lambda_{3}\left[\Lambda_{1} \Lambda_{3}\right]^{\dagger}
$$

satisfies the identity

$$
L_{1,3}^{2}=\left[N_{1}^{2}+\tilde{N}_{1}^{2}\right] L_{1,3}=0
$$


when $T=0$ and since $L_{1,3}$ is the square of a hermitian operator of the form $O O^{\dagger}$, it entails that $O$ itself is zero:

$$
\Lambda_{1} \Lambda_{3}=0
$$

when $T=0$. Similarly one finds the identities (valid when $T=0$ ):

$$
N_{1}\left[\Lambda_{1} \Lambda_{1}^{\dagger}+\Lambda_{3} \Lambda_{3}^{\dagger}\right]=\tilde{N}_{1}\left[\Lambda_{3} \Lambda_{1}^{\dagger}-\Lambda_{1} \Lambda_{3}^{\dagger}\right]
$$

and

$$
N_{1}\left[\Lambda_{1}^{\dagger} \Lambda_{1}+\Lambda_{3}^{\dagger} \Lambda_{3}\right]=\tilde{N}_{1}\left[\Lambda_{1}^{\dagger} \Lambda_{3}-\Lambda_{3}^{\dagger} \Lambda_{1}\right]
$$

A similar argument works for $L_{2,4}$ etc. and we note from 75 that $L_{1,3}$ and $L_{2,4}$ commute.

\subsection{The Projection Operator $\Pi_{T=0}$}

We will not need this explicitly, but we indicate briefly how to construct it. What one does is write $N_{1}$ and $\tilde{N}_{1}$ in the form:

$$
N_{1}=f_{i} f_{i}^{\dagger}
$$

and

$$
\tilde{N}_{1}=f_{i} \varepsilon_{i j} f_{j}^{\dagger}
$$

and then one sees that:

$$
T=N_{1}^{2}+\tilde{N}_{1}^{2}=N_{0,2}+N_{2,0}
$$

where we define:

$$
\begin{gathered}
N_{k, p}=\left(f_{i_{1}} \ldots f_{i_{k}}\right)\left(f_{j_{1}} \ldots f_{j_{p}}\right) \\
{\left[\varepsilon_{j_{1} l_{1}} \ldots \varepsilon_{j_{p} l_{p}}\right]\left(f_{l_{1}} \ldots f_{l_{p}}\right)^{\dagger}\left(f_{i_{1}} \ldots f_{i_{k}}\right)^{\dagger} .}
\end{gathered}
$$

Then the projection operator has the form:

$$
\Pi_{T=0}=\sum_{k, p=0}^{\infty} a_{k, p} N_{k, p},
$$

where the coefficients can be calculated by the requirement:

$$
T \Pi_{T=0}=0
$$

plus the requirement that the operator be the maximal projection operator that satisfies these equations. There is no doubt a more elegant way to write this, if the need arises.

\subsection{The Projection Operator $\Pi_{Q=0}$}

In order to construct the projection operator for the subspace $Q=0$, we proceed as follows: The operator that occurs in Sect. 7 can be written in the useful form:

$$
Q=c_{i} c_{j}^{\dagger}\left\{\Lambda_{i}, \Lambda_{j}^{\dagger}\right\}=N_{1} N_{1}^{\prime}-\tilde{N}_{1} \tilde{N}_{1}^{\prime},
$$


and we also define the operator

$$
Q_{5}=c_{k}\left(\gamma_{5}\right)_{k i} c_{j}^{\dagger}\left\{\Lambda_{i}, \Lambda_{j}^{\dagger}\right\}=N_{1} \tilde{N}_{1}^{\prime}+\tilde{N}_{1} N_{1}^{\prime},
$$

where we define:

$$
\begin{gathered}
N_{1}^{\prime}=c_{i} c_{i}^{\dagger}, \\
\tilde{N}_{1}^{\prime}=c_{i}\left(\gamma_{5}\right)_{i j} c_{j}^{\dagger} .
\end{gathered}
$$

If we assume that we are already in the subspace generated by $\Pi_{\Lambda_{i}=0}$, then it is easy to see that we require the equations:

$$
\begin{gathered}
c_{i}^{\dagger} \Lambda_{i}^{\dagger} E_{1}=0, \\
\Lambda_{j} E_{1}=0,
\end{gathered}
$$

and that these imply the equations:

$$
Q E_{1}=Q_{5} E_{1}=0,
$$

which in turn imply the relations:

$$
\begin{aligned}
& T N_{1}^{\prime} E_{1}=0, \\
& T^{\prime} N_{1} E_{1}=0,
\end{aligned}
$$

and this means that we can write:

$$
\begin{aligned}
\Pi_{Q=0,(\Lambda=0)}=\left\{\Pi_{N_{1}=0}+\Pi_{N_{1}>0}\left[\Pi_{N_{1}^{\prime}=0} \Pi_{\Lambda=0}\right.\right. \\
\left.\left.+\Pi_{N_{1}^{\prime}>0} \Pi_{T=0} \Pi_{T^{\prime}=0} \Pi_{\Lambda=0,(T=0)}\right] \frac{1}{2}\left[1+\frac{\tilde{N}_{1} \tilde{N}_{1}^{\prime}}{N_{1} N_{1}^{\prime}}\right]\right\},
\end{aligned}
$$

and this is the relevant projection operator.

Let us now introduce the abbreviations:

$$
\begin{gathered}
\mathscr{J}=\frac{\tilde{N}_{1}}{N_{1}}, \\
\mathscr{J}^{\prime}=\frac{\tilde{N}_{1}^{\prime}}{N_{1}^{\prime}},
\end{gathered}
$$

which are meant to summarize the fact that these operators act like the square root of $(-1)$ in our Fock space once it has been projected onto $E_{1} . \mathscr{J}$ and $\mathscr{J}^{\prime}$ will frequently appear in this role below. We also note that

$$
\mathscr{J} \Pi_{1}=-\mathscr{J}^{\prime} \Pi_{1} .
$$

\subsection{The Projection Operator $\Pi_{T^{\prime}=0}$}

It can be verified using the Fierz transformations that the following identity holds:

$$
T^{\prime}=\left(N_{1}^{\prime}\right)^{2}+\left(\tilde{N}_{1}^{\prime}\right)^{2}=\frac{1}{2} v_{\mu} v_{\mu}^{\dagger},
$$

where we use the notation:

$$
v_{\mu}=\bar{c} \gamma_{\mu} c
$$


Now we observe the identity:

$$
v_{\mu}^{\dagger} v_{v}=8 \delta_{\mu \nu}\left[1+N_{1}^{\prime}\right]+4 \bar{c} \gamma^{\mu} \gamma_{0} \gamma^{\nu} c^{\dagger}+v_{\nu} v_{\mu}^{\dagger},
$$

and using this we see that the projection operator can be written:

where

$$
\Pi_{T^{\prime}=0}=\sum_{k=0}^{\infty} a_{2 k} M_{2 k}
$$

$$
\begin{gathered}
M_{0}=1, \\
M_{2 k}=\left[v_{\mu_{1}} v_{\mu_{2}} \ldots v_{\mu_{k}}\right]\left[v_{\mu_{1}} v_{\mu_{2}} \ldots v_{\mu_{k}}\right]^{\dagger} \quad(k \geqq 1),
\end{gathered}
$$

and

$$
\begin{gathered}
a_{0}=1 \\
a_{2 k}=\frac{(-1)^{k}}{8^{k} k !\left[N_{1}^{\prime}-k\right]\left[N_{1}^{\prime}-k+1\right] \ldots\left[N_{1}^{\prime}-1\right]} .
\end{gathered}
$$

\subsection{The Projection Operator $\Pi_{N_{1}=0}$}

The construction of this operator was given in [2] but will be repeated here for completeness:

$$
\Pi_{N_{1}=0}=\sum_{k=0}^{\infty} \frac{(-1)^{k}}{k !} N_{k},
$$

where for any relevant set of fields $\phi_{i}$ and their adjoints $\phi_{i}^{\dagger}$,

$$
\begin{gathered}
N_{1}=\phi_{i} \phi_{i}^{\dagger}, \\
N_{k}=\left[\phi_{i_{1}} \phi_{i_{2}} \ldots \phi_{i_{k}}\right]\left[\phi_{i_{1}} \phi_{i_{2}} \ldots \phi_{i_{k}}\right]^{\dagger} .
\end{gathered}
$$

It is easy to see that:

$$
N_{1} N_{k}=k N_{k}+N_{k+1},
$$

and this enables one to show that

$$
N_{1} \Pi_{N_{1}=0}=0 \text {, }
$$

which is the desired property for the operator.

9.8. The Projection Operator $\Pi_{N_{1}}=q$

This is easily constructed from the above:

$$
\Pi_{N_{1}=q}=\left[\phi_{i_{1}} \phi_{i_{2}} \ldots \phi_{i_{q}}\right] \Pi_{N_{1}=0}\left[\phi_{i_{1}} \phi_{i_{2}} \ldots \phi_{i_{q}}\right]^{\dagger},
$$

and it satisfies

$$
N_{1} \Pi_{N_{1}=q}=q \Pi_{N_{1}=q}
$$

Let us also note that

$$
\phi_{i} \Pi_{N_{1}=q}=\Pi_{N_{1}=(q+1)} \phi_{i}
$$


and

$$
\Pi_{N_{1}>q}=\sum_{k=q+1}^{\infty} \Pi_{N_{1}=k}
$$

9.9. The Operator $1 / N_{1}$

Frequently we need the operator

$$
\frac{1}{N_{1}}=\sum_{k=1}^{\infty} a_{k} N_{k}
$$

which satisfies the relation:

$$
\frac{1}{N_{1}} N_{1}=1-\Pi_{N_{1}=0}=\Pi_{N_{1}>0} .
$$

This set of equations define the coefficients $a_{k}$. The first few values are:

$$
\begin{gathered}
a_{1}=1, \\
a_{2}=-\frac{3}{4}, \\
a_{3}=\frac{11}{36}, \\
a_{4}=-\frac{25}{288} .
\end{gathered}
$$

We do not need the general form, or indeed any of the coefficients for present purposes. They are given here just for an example of how things work. In practice we leave this operator in the form $\frac{1}{N_{1}}$ which is easiest to work with. It should be noted that if one does not think carefully, it is easy to start generating this series (usually mixed in with other things) without realizing what it is, and that can lead to considerable obfuscation of simple results.

\subsection{The Projection Operator for the Subspace where $\partial_{\mu} \partial_{\mu}^{\dagger}=0$}

One of the equations for the subspace $\Pi_{1}$ is:

$$
\partial_{\mu}^{\dagger} \Pi_{1}=0
$$

and the projection operator that satisfies this equation is:

$$
\Pi_{\partial^{\dagger}=0}=1-\frac{1}{N_{1}} \partial_{\mu} \partial_{\mu}^{\dagger}+\frac{1}{N_{1}^{2}} \partial_{\mu} \partial_{\nu} \partial_{\nu}^{\dagger} \partial_{\mu}^{\dagger}+\ldots,
$$

which in full is:

$$
\Pi_{\partial^{\dagger}=0}=\sum_{k=0}^{\infty}\left\{\frac{-1}{N_{1}}\right\}^{k} \partial_{\mu_{1}} \partial_{\mu_{2}} \ldots \partial_{\mu_{k}} \partial_{\mu_{k}}^{\dagger} \ldots \partial_{\mu_{2}}^{\dagger} \partial_{\mu_{1}}^{\dagger},
$$

where the term with $k=0$ is defined to be 1 . This is easily seen to satisfy the Eq. (135) using the identity:

$$
\left[\partial_{\mu}^{\dagger}, \partial_{v}\right]=\delta_{\mu v} N_{1},
$$

which was used in [2] and also to establish 26. 


\subsection{The Projection Operator $\Pi_{\varepsilon}$}

The orthogonal projection operator that projects onto the space of polynomials proportional to $T_{\varepsilon}^{4}$ is:

$$
\Pi_{\varepsilon}=T_{\varepsilon}^{4}\left[T_{\varepsilon}^{4}\right]^{\dagger} .
$$

\subsection{The Total Projection Operator $\Pi_{1}=\Pi_{2}$}

We have now constructed all the operators necessary to project out all the solutions of the space $E_{1}$. This total operator is:

$$
\begin{aligned}
\Pi_{1}= & \Pi_{N_{1}=0}+\Pi_{N_{1}>0} \Pi_{\varepsilon=0} \Pi_{\partial^{\dagger}=0} \\
& \times\left\{\Pi_{N_{1}^{\prime}=0}\left[\Pi_{T=0} \Pi_{\Lambda=0,(T=0)}+\Pi_{T>0} \Pi_{\Lambda=0,(T>0)}\right]\right. \\
& \left.+\Pi_{N^{\prime}>0} \Pi_{T=0} \Pi_{T^{\prime}=0} \Pi_{\Lambda=0,(T=0)} \Pi_{Q=0,\left(\Lambda=T=T^{\prime}=0\right)}\right\},
\end{aligned}
$$

and the equation:

$$
\Pi_{1}=\Pi_{2}
$$

holds because the operator $d_{1}$ must be zero as a consequence of the fact that $\delta_{1}$ is not present in the decomposition of $\delta$ in Eq. (23), so that

$$
d_{1}=\Pi_{1} \delta_{1} \Pi_{1}=0 \text {. }
$$

Now we are ready to proceed to the calculation of $d_{2}, \Delta_{2}$ and the spaces $E_{3}$.

\section{Part III. The Space $E_{3}=E_{\text {o }}$}

\section{The Operator $d_{2}$}

The operator $\delta_{2}$ was written out in Eq. (25) above and the operator $d_{2}$ is defined by:

$$
d_{2}=\Pi_{2} \delta_{2} \Pi_{2} \text {. }
$$

From the previous section we know the form of $\Pi_{2}$. It is useful to note the following identity at this point:

$$
\left[\delta_{2}, \tilde{N}_{1}\right]=0
$$

and indeed the more general equation:

$$
\left[\delta, \tilde{N}_{1}\right]=0
$$

also holds for the complete operator defined in 12 .

The following identities are simple to establish by calculation:

$$
\begin{gathered}
\left\{\nabla_{i}, \Lambda_{j}^{\dagger}\right\}=0, \\
{\left[\partial_{\mu}^{\dagger}, \nabla_{i}\right]=\left(\gamma_{0} \gamma^{\mu}\right)_{i j} \Lambda_{j}^{\dagger},} \\
{\left[\partial_{\mu}, \Lambda_{i}\right]=0,} \\
{\left[\partial_{\mu}, \Lambda_{i}^{\dagger}\right]=0,} \\
{\left[\partial_{\mu}, \nabla_{i}\right]=0,} \\
{\left[\Lambda_{i}, \nabla_{j}\right]=\left(\gamma_{0} \gamma^{\mu}\right)_{i j} \partial_{\mu}+\left(\gamma_{0} \gamma^{\mu} \gamma_{5}\right)_{i j} \tilde{\partial}_{\mu},}
\end{gathered}
$$


where we define the new operator $\tilde{\partial}_{\mu}$ by:

$$
\begin{aligned}
\tilde{\partial}_{\mu}= & \sum_{k=0}^{\infty} \frac{1}{k !}\left\{A_{\mu_{1} \mu_{2} \ldots \mu_{k} \mu} B_{\mu_{1} \mu_{2} \ldots \mu_{k}}^{\dagger}-B_{\mu_{1} \mu_{2} \ldots \mu_{k} \mu} A_{\mu_{1} \mu_{2} \ldots \mu_{k}}^{\dagger}\right. \\
& +G_{\mu_{1} \mu_{2} \ldots \mu_{k} \mu} F_{\mu_{1} \mu_{2} \ldots \mu_{k}}^{\dagger}-F_{\mu_{1} \mu_{2} \ldots \mu_{k} \mu} G_{\mu_{1} \mu_{2} \ldots \mu_{k}}^{\dagger} \\
& \left.+\psi_{i \mu_{1} \mu_{2} \ldots \mu_{k} \mu}\left(\gamma_{5}\right)_{i j} \psi_{j \mu_{1} \mu_{2} \ldots \mu_{k}}^{\dagger}\right\}
\end{aligned}
$$

The following equations are also easy to establish by calculation:

$$
\begin{gathered}
{\left[\Lambda_{i}, \widetilde{\partial}_{\mu}\right]=0,} \\
{\left[\Lambda_{i}^{\dagger}, \widetilde{\partial}_{\mu}\right]=0,} \\
{\left[\tilde{\partial}_{\mu}^{\dagger}, \nabla_{i}\right]=\left(\gamma_{0} \gamma^{\mu} \gamma_{5}\right)_{i j} \Lambda_{j}^{\dagger},} \\
{\left[\widetilde{\partial}_{\mu}, \nabla_{i}\right]=0,} \\
{\left[\partial_{\mu}^{\dagger}, \widetilde{\partial}_{v}\right]=\delta_{\mu v} \tilde{N}_{1},} \\
{\left[\widetilde{\partial}_{\mu}^{\dagger}, \widetilde{\partial}_{v}\right]=\delta_{\mu \nu} N_{1},}
\end{gathered}
$$

and together with the form of $\Pi_{\Lambda=0}$ given above and the following equations:

$$
\begin{aligned}
& \Pi_{2} \Lambda_{i}^{\dagger}=0, \\
& \Pi_{2} \partial_{\mu}=0,
\end{aligned}
$$

which are the adjoint of equations found above (since $\Pi_{1}=\Pi_{2}$ ), we see that

$$
\begin{aligned}
d_{2}= & \Pi_{2}\left[c_{i} \nabla_{i}-\bar{c} \gamma_{\mu} c \varepsilon_{\mu}^{\dagger}\right] \Pi_{2}=\Pi_{N_{1}=0}\left(-\bar{c} \gamma_{\mu} c \varepsilon_{\mu}^{\dagger}\right) \\
& +\Pi_{N_{1}>0} \Pi_{\varepsilon=0} \Pi_{\partial^{\dagger}=0} \Pi_{N_{1}^{\prime}>0} \Pi_{T=0} \Pi_{T^{\prime}=0} \Pi_{\Lambda=0,(T=0)} \Pi_{Q=0,\left(\Lambda=T=T^{\prime}=0\right.} c_{i} \nabla_{i}
\end{aligned}
$$

which can also be written:

$$
\begin{aligned}
d_{2}= & \Pi_{N_{1}=0}\left(-\bar{c} \gamma_{\mu} c \varepsilon_{\mu}^{\dagger}\right) \\
& +\Pi_{\Lambda=0,(T=0)} \nabla_{i} \Pi_{Q=0,\left(\Lambda=T=T^{\prime}=0\right)} \Pi_{T^{\prime}=0} c_{i} \Pi_{N_{1}>0} \Pi_{\varepsilon=0} \Pi_{\partial^{\dagger}=0} \Pi_{T=0} .
\end{aligned}
$$

In writing the above, the following identities are useful:

$$
\begin{gathered}
\Pi_{2} c_{i} \Pi_{2}=\Pi_{2} c_{i}=\Pi_{Q=0,\left(\Lambda=T=T^{\prime}=0\right)} \Pi_{T^{\prime}=0} c_{i} \Pi_{2} \\
=\frac{1}{2}\left[c_{i}-\frac{\tilde{N}_{1}}{N_{1}}\left(\gamma_{5}\right)_{i j} c_{j}\right] \Pi_{2}, \\
\Pi_{2} \nabla_{i} \Pi_{2}=\Pi_{2} \nabla_{i}=\Pi_{\Lambda=0} \nabla_{i} \Pi_{2}=\left\{\nabla_{i}-\frac{1}{2 N_{1}}\left[\left(\gamma_{0} \gamma^{\mu}\right)_{i j} \partial_{\mu}-\left(\gamma_{0} \gamma^{\mu} \gamma_{5}\right)_{i j} \widetilde{\partial}_{\mu}\right] \Lambda_{j}^{\dagger}\right\} \Pi_{2}
\end{gathered}
$$

as are their adjoints:

$$
\begin{gathered}
\Pi_{2} c_{i}^{\dagger} \Pi_{2}=c_{i}^{\dagger} \Pi_{2}=\Pi_{2} \frac{1}{2}\left[c_{i}^{\dagger}+\frac{\tilde{N}_{1}}{N_{1}}\left(\gamma_{5}\right)_{i j} c_{j}^{\dagger}\right], \\
\Pi_{2} \nabla_{i}^{\dagger} \Pi_{2}=\nabla_{i}^{\dagger} \Pi_{2}=\Pi_{2} \nabla_{i}^{\dagger} \Pi_{\Lambda=0} \\
=\Pi_{2}\left\{\nabla_{i}^{\dagger}-\frac{1}{2 N_{1}}\left[\left(\gamma_{0} \gamma^{\mu}\right)_{i j} \partial_{\mu}^{\dagger}-\left(\gamma_{0} \gamma^{\mu} \gamma_{5}\right)_{i j} \tilde{\partial}_{\mu}^{\dagger}\right] \Lambda_{j}\right\} .
\end{gathered}
$$


In the foregoing, we have used the identity:

$$
\Pi_{\Lambda=0} \Pi_{\partial^{\dagger}=0} \nabla_{i} \Pi_{2}=\Pi_{\Lambda=0} \nabla_{i} \Pi_{2}
$$

which is a consequence of (147). From now on we will forget about the sector where $N_{1}=0$ since it is of no interest at present.

\section{First Expression for $\Delta_{2}$}

Given the foregoing the computation of $\Delta_{2}$ yields:

$$
\Delta_{2}=\Pi_{2}\left[\left\{\nabla_{i}, \nabla_{j}^{\dagger}\right\} c_{i} c_{j}^{\dagger}+\nabla_{j}^{\dagger} \Pi_{\Lambda=0} \nabla_{i} P_{j i}^{+}-8 N_{1}^{\prime}\right] \Pi_{2},
$$

where we define the "chiral projectors":

$$
P_{i j}^{+}=\frac{1}{2}\left[\delta_{i j}+\mathscr{J}\left(\gamma_{5}\right)_{i j}\right]
$$

and

$$
P_{i j}^{-}=\frac{1}{2}\left[\delta_{i j}-\mathscr{J}\left(\gamma_{5}\right)_{i j}\right] .
$$

To derive this we need also to note that as a consequence of Eqs. (29)-(33):

$$
\widetilde{\partial}_{\mu}^{\dagger} c_{i}^{\dagger} \Pi_{2}=-\partial_{\mu}^{\dagger}\left(\gamma_{5}\right)_{i j} c_{j}^{\dagger} \Pi_{2}=0
$$

\section{Evaluation of the Anticommutator}

It is straightforward to evaluate the expression:

$$
\begin{aligned}
& c_{i} c_{j}^{\dagger}\left\{\nabla_{i}, \nabla_{j}^{\dagger}\right\}=\sum_{k=0}^{\infty} \frac{1}{k !}\left\{\left[\left(\gamma^{\mu}\right)_{p i} c_{i} A_{\mu \mu_{1} \mu_{2} \ldots \mu_{k}}+\left(\gamma^{\mu} \gamma_{5}\right)_{p i} c_{i} B_{\mu \mu_{1} \mu_{2} \ldots \mu_{k}}\right]\right. \\
& \times\left[\left(\gamma^{v}\right)_{p j} c_{j} A_{v \mu_{1} \mu_{2} \ldots \mu_{k}}+\left(\gamma^{v} \gamma_{5}\right)_{p j} c_{j} B_{v \mu_{1} \mu_{2} \ldots \mu_{k}}\right]^{\dagger} \\
& +\left[\bar{\psi}_{\mu_{1} \mu_{2} \ldots \mu_{k}} c\right]\left[\bar{\psi}_{\mu_{1} \mu_{2} \ldots \mu_{k}} c\right]^{\dagger} \\
& -\left[\bar{\psi}_{\mu_{1} \mu_{2} \ldots \mu_{k}} \gamma_{5} c\right]\left[\bar{\psi}_{\mu_{1} \mu_{2} \ldots \mu_{k}} \gamma_{5} c\right]^{\dagger} \\
& +\frac{1}{2}\left[\bar{\psi}_{\mu_{1} \mu_{2} \ldots \mu_{k}} \gamma_{\sigma} c\right]\left[\bar{\psi}_{\mu_{1} \mu_{2} \ldots \mu_{k}} \gamma_{\sigma} c\right]^{\dagger} \\
& +\frac{1}{2}\left[\bar{\psi}_{\mu_{1} \mu_{2} \ldots \mu_{k}} \gamma_{\sigma} \gamma_{5} c\right]\left[\bar{\psi}_{\mu_{1} \mu_{2} \ldots \mu_{k}} \gamma_{\sigma} \gamma_{5} c\right]^{\dagger} \\
& +k \frac{3}{4}\left[\bar{\psi}_{\mu \mu_{2} \ldots \mu_{k}} \gamma^{\mu} c\right]\left[\bar{\psi}_{v \mu_{2} \ldots \mu_{k}} \gamma^{v} c\right]^{\dagger} \\
& +\frac{3}{4}\left[\bar{\psi}_{\mu \mu_{2} \ldots \mu_{k}} \gamma^{\mu} \gamma_{5} c\right]\left[\bar{\psi}_{v \mu_{2} \ldots \mu_{k}} \gamma^{v} \gamma_{5} c\right]^{\dagger} \\
& +\frac{1}{4}\left[\bar{\psi}_{\mu \mu_{2} \ldots \mu_{k}} c+\bar{\psi}_{\sigma \mu_{2} \ldots \mu_{k}} \gamma_{\mu}{ }^{\sigma} c\right]\left[\bar{\psi}_{\mu \mu_{2} \ldots \mu_{k}} c+\bar{\psi}_{\sigma \mu_{2} \ldots \mu_{k}} \gamma_{\mu}{ }^{\sigma} c\right]^{\dagger} \\
& -\frac{1}{4}\left[\bar{\psi}_{\mu \mu_{2} \ldots \mu_{k}} \gamma_{5} c+\bar{\psi}_{\sigma \mu_{2} \ldots \mu_{k}} \gamma_{\mu}{ }^{\sigma} \gamma_{5} c\right]\left[\bar{\psi}_{\mu \mu_{2} \ldots \mu_{k}} \gamma_{5} c+\bar{\psi}_{\sigma \mu_{2} \ldots \mu_{k}} \gamma_{\mu}{ }^{\sigma} \gamma_{5} c\right]^{\dagger} \\
& +\frac{1}{8}\left[\bar{\psi}_{\mu \mu_{2} \ldots \mu_{k}} \gamma_{v} c-\bar{\psi}_{v \mu_{2} \ldots \mu_{k}} \gamma_{\mu} c-\varepsilon_{\mu \nu \lambda \sigma} \bar{\psi}_{\mu_{2} \ldots \mu_{k}}^{\sigma} \gamma^{\lambda} \gamma_{5} c\right] \\
& \times\left[\bar{\psi}_{\mu \mu_{2} \ldots \mu_{k}} \gamma_{\nu} c-\bar{\psi}_{\nu \mu_{2} \ldots \mu_{k}} \gamma_{\mu} c-\varepsilon_{\mu \nu \varrho \kappa} \bar{\psi}_{\mu_{2} \ldots \mu_{k}}^{\varrho} \gamma^{\kappa} \gamma_{5} c\right]^{\dagger} \\
& \left.+4\left[c_{i} F_{\mu_{1} \mu_{2} \ldots \mu_{k}}-\left(\gamma_{5}\right)_{i j} c_{j} G_{\mu_{1} \mu_{2} \ldots \mu_{k}}\right]\left[c_{i} F_{\mu_{1} \mu_{2} \ldots \mu_{k}}-\left(\gamma_{5}\right)_{i l} c_{l} G_{\mu_{1} \mu_{2} \ldots \mu_{k}}\right]^{\dagger}\right) \\
& +k\left[c_{i}\left(\gamma_{0} \gamma^{\mu}\right)_{i p} F_{\mu \mu_{2} \ldots \mu_{k}}+c_{i}\left(\gamma_{0} \gamma^{\mu} \gamma_{5}\right)_{i p} G_{\mu \mu_{2} \ldots \mu_{k}}\right] \\
& \left.\times\left[c_{i}\left(\gamma_{0} \gamma^{\mu}\right)_{i p} F_{\mu \mu_{2} \ldots \mu_{k}}+c_{i}\left(\gamma_{0} \gamma^{\mu} \gamma_{5}\right)_{i p} G_{\mu \mu_{2} \ldots \mu_{k}}\right]^{\dagger}\right\} \text {. }
\end{aligned}
$$


Note that some of the above terms occur with negative coefficients. However all of these disappear using the properties of $\Pi_{1}$, when this expression is placed between two operators $\Pi_{1}$, which is the next step.

\section{Second Expression for $\boldsymbol{\Delta}_{2}$}

Using the above and the properties of $\Pi_{2}$ we get

$$
\begin{aligned}
\Delta_{2}= & \Pi_{2}\left\{\nabla_{j}^{\dagger} \Pi_{\Lambda=0} \nabla_{i} P_{j i}^{+}+\sum_{k=0}^{\infty} \frac{1}{k !}\left[\left[\left(\gamma^{\mu}\right)_{p i} c_{i} A_{\mu \mu_{1} \mu_{2} \ldots \mu_{k}}+\left(\gamma^{\mu} \gamma_{5}\right)_{p i} c_{i} B_{\mu \mu_{1} \mu_{2} \ldots \mu_{k}}\right]\right.\right. \\
& \times\left[\left(\gamma^{v}\right)_{p j} c_{j} A_{v \mu_{1} \mu_{2} \ldots \mu_{k}}+\left(\gamma^{v} \gamma_{5}\right)_{p j} c_{j} B_{v \mu_{1} \mu_{2} \ldots \mu_{k}}\right]^{\dagger} \\
& +\frac{1}{2}\left[\bar{\psi}_{\mu_{1} \mu_{2} \ldots \mu_{k}} \gamma_{\sigma} c\right]\left[\bar{\psi}_{\mu_{1} \mu_{2} \ldots \mu_{k}} \gamma_{\sigma} c\right]^{\dagger} \\
& +\frac{1}{2}\left[\bar{\psi}_{\mu_{1} \mu_{2} \ldots \mu_{k}} \gamma_{\sigma} \gamma_{5} c\right]\left[\bar{\psi}_{\mu_{1} \mu_{2} \ldots \mu_{k}} \gamma_{\sigma} \gamma_{5} c\right]^{\dagger} \\
& +k \frac{3}{4}\left[\bar{\psi}_{\mu \mu_{2} \ldots \mu_{k}} \gamma^{\mu} c\right]\left[\bar{\psi}_{v \mu_{2} \ldots \mu_{k}} \gamma^{v} c\right]^{\dagger} \\
& +\frac{3}{4}\left[\bar{\psi}_{\mu \mu_{2} \ldots \mu_{k}} \gamma^{\mu} \gamma_{5} c\right]\left[\bar{\psi}_{v \mu_{2} \ldots \mu_{k}} \gamma^{v} \gamma_{5} c\right]^{\dagger} \\
& +\frac{1}{8}\left[\bar{\psi}_{\mu \mu_{2} \ldots \mu_{k}} \gamma_{v} c-\bar{\psi}_{v \mu_{2} \ldots \mu_{k}} \gamma_{\mu} c-\varepsilon_{\mu v \lambda \sigma} \bar{\psi}_{\mu_{2} \ldots \mu_{k}}^{\sigma} \gamma^{\lambda} \gamma_{5} c\right] \\
& \times\left[\bar{\psi}_{\mu \mu_{2} \ldots \mu_{k}} \gamma_{v} c-\bar{\psi}_{v \mu_{2} \ldots \mu_{k}} \gamma_{\mu} c-\varepsilon_{\mu v \varrho \kappa} \bar{\psi}_{\mu_{2} \ldots \mu_{k}} \gamma^{\kappa} \gamma_{5} c\right]^{\dagger} \\
& +4\left[c_{i} F_{\mu_{1} \mu_{2} \ldots \mu_{k}}-\left(\gamma_{5}\right)_{i j} c_{j} G_{\mu_{1} \mu_{2} \ldots \mu_{k}}\right]\left[c_{i} F_{\mu_{1} \mu_{2} \ldots \mu_{k}}-\left(\gamma_{5}\right)_{i l} c_{l} G_{\mu_{1} \mu_{2} \ldots \mu_{k}}\right]^{\dagger} \\
& +k\left[c_{i}\left(\gamma_{0} \gamma^{\mu}\right)_{i p} F_{\mu \mu_{2} \ldots \mu_{k}}+c_{i}\left(\gamma_{0} \gamma^{\mu} \gamma_{5}\right)_{i p} G_{\mu \mu_{2} \ldots \mu_{k}}\right] \\
& \left.\left.\times\left[c_{j}\left(\gamma_{0} \gamma^{v}\right)_{j p} F_{v \mu_{2} \ldots \mu_{k}}+c_{i}\left(\gamma_{0} \gamma^{v} \gamma_{5}\right)_{j p} G_{v \mu_{2} \ldots \mu_{k}}\right]^{\dagger}\right]-8 N_{1}^{\prime}\right\} \Pi_{2} .
\end{aligned}
$$

Note that this operator is still not in manifestly non-negative form. If the last term preceded by the - sign were not present it would be easy to deduce that no $c$ dependent terms survive in $E_{3}=\operatorname{Ker} \Delta_{2}$. However $c$-dependent terms are in fact present, and in a sense that is why this form fails to be manifestly non-negative.

\section{Second Form for the Anticommutator}

The relevant anticommutator at this stage can also be written in the form:

$$
\left\{\nabla_{i}, \nabla_{j}^{\dagger}\right\}=\delta_{i j} M+\left(\gamma_{5}\right)_{i j} \tilde{M}+\frac{1}{2}\left(\gamma_{\mu \nu}\right)_{i j} L_{\mu \nu}+\frac{1}{2}\left(\gamma_{\mu \nu} \gamma_{5}\right)_{i j} \tilde{L}_{\mu \nu},
$$

where the operators are defined as follows:

$$
\begin{aligned}
M= & \sum_{k=0}^{\infty} \frac{1}{k !}\left\{k\left[\left(A_{\mu_{1} \mu_{2} \ldots \mu_{k}}\right)\left(A_{\mu_{1} \mu_{2} \ldots \mu_{k}}\right)^{\dagger}+\left(B_{\mu_{1} \mu_{2} \ldots \mu_{k}}\right)\left(B_{\mu_{1} \mu_{2} \ldots \mu_{k}}\right)^{\dagger}\right]\right. \\
& +(2+k)\left(\psi_{\mu_{1} \mu_{2} \ldots \mu_{k}}\right)\left(\psi_{\mu_{1} \mu_{2} \ldots \mu_{k}}\right)^{\dagger} \\
& \left.+(4+k)\left[\left(F_{\mu_{1} \mu_{2} \ldots \mu_{k}}\right)\left(F_{\mu_{1} \mu_{2} \ldots \mu_{k}}\right)^{\dagger}+\left(G_{\mu_{1} \mu_{2} \ldots \mu_{k}}\right)\left(G_{\mu_{1} \mu_{2} \ldots \mu_{k}}\right)^{\dagger}\right]\right\},
\end{aligned}
$$

and

$$
\begin{aligned}
\tilde{M}= & \sum_{k=0}^{\infty} \frac{1}{k !}\left\{k\left[\left(A_{\mu_{1} \mu_{2} \ldots \mu_{k}}\right)\left(B_{\mu_{1} \mu_{2} \ldots \mu_{k}}\right)^{\dagger}-\left(B_{\mu_{1} \mu_{2} \ldots \mu_{k}}\right)\left(A_{\mu_{1} \mu_{2} \ldots \mu_{k}}\right)^{\dagger}\right]\right. \\
& +(2+k)\left(\psi_{i \mu_{1} \mu_{2} \ldots \mu_{k}}\right)\left(\gamma_{5}\right)_{i j}\left(\psi_{j \mu_{1} \mu_{2} \ldots \mu_{k}}\right)^{\dagger} \\
& \left.+(4+k)\left[\left(G_{\mu_{1} \mu_{2} \ldots \mu_{k}}\right)\left(F_{\mu_{1} \mu_{2} \ldots \mu_{k}}\right)^{\dagger}-\left(F_{\mu_{1} \mu_{2} \ldots \mu_{k}}\right)\left(G_{\mu_{1} \mu_{2} \ldots \mu_{k}}\right)^{\dagger}\right]\right\}
\end{aligned}
$$


and:

$$
\begin{aligned}
L_{\mu \nu}= & \sum_{k=0}^{\infty} \frac{1}{k !}\left\{\left(A_{\mu \mu_{1} \mu_{2} \ldots \mu_{k}}\right)\left(A_{\mu_{1} \mu_{2} \ldots \mu_{k}}^{v}\right)^{\dagger}-\left(A_{v \mu_{1} \mu_{2} \ldots \mu_{k}}\right)\left(A_{\mu_{1} \mu_{2} \ldots \mu_{k}}^{\mu}\right)^{\dagger}\right. \\
& +\left(B_{\mu \mu_{1} \mu_{2} \ldots \mu_{k}}\right)\left(B_{\mu_{1} \mu_{2} \ldots \mu_{k}}^{v}\right)^{\dagger}-\left(B_{v \mu_{1} \mu_{2} \ldots \mu_{k}}\right)\left(B_{\mu_{1} \mu_{2} \ldots \mu_{k}}^{\mu}\right)^{\dagger} \\
& +\left(\psi_{\mu \mu_{1} \mu_{2} \ldots \mu_{k}}\right)\left(\psi_{\mu_{1} \mu_{2} \ldots \mu_{k}}^{v}\right)^{\dagger}-\left(\psi_{v \mu_{1} \mu_{2} \ldots \mu_{k}}\right)\left(\psi_{\mu_{1} \mu_{2} \ldots \mu_{k}}^{\mu}\right)^{\dagger} \\
& +\left(F_{\mu \mu_{1} \mu_{2} \ldots \mu_{k}}\right)\left(F_{\mu_{1} \mu_{2} \ldots \mu_{k}}^{v}\right)^{\dagger}-\left(F_{v \mu_{1} \mu_{2} \ldots \mu_{k}}\right)\left(F_{\mu_{1} \mu_{2} \ldots \mu_{k}}^{\mu}\right)^{\dagger} \\
& \left.+\left(G_{\mu \mu_{1} \mu_{2} \ldots \mu_{k}}\right)\left(G_{\mu_{1} \mu_{2} \ldots \mu_{k}}^{v}\right)^{\dagger}-\left(G_{v \mu_{1} \mu_{2} \ldots \mu_{k}}\right)\left(G_{\mu_{1} \mu_{2} \ldots \mu_{k}}^{\mu}\right)^{\dagger}\right\}
\end{aligned}
$$

and:

$$
\begin{aligned}
\tilde{L}_{\mu \nu}= & \sum_{k=0}^{\infty} \frac{1}{k !}\left(A_{\mu \mu_{1} \mu_{2} \ldots \mu_{k}}\right)\left(B_{\mu_{1} \mu_{2} \ldots \mu_{k}}^{v}\right)^{\dagger}-\left(A_{v \mu_{1} \mu_{2} \ldots \mu_{k}}\right)\left(B_{\mu_{1} \mu_{2} \ldots \mu_{k}}^{\mu}\right)^{\dagger} \\
& +\left(B_{\mu \mu_{1} \mu_{2} \ldots \mu_{k}}\right)\left(A_{\mu_{1} \mu_{2} \ldots \mu_{k}}^{v}\right)^{\dagger}-\left(B_{v \mu_{1} \mu_{2} \ldots \mu_{k}}\right)\left(A_{\mu_{1} \mu_{2} \ldots \mu_{k}}^{\mu}\right)^{\dagger} \\
& +\left(\psi_{i \mu \mu_{1} \mu_{2} \ldots \mu_{k}}\right)\left(\gamma_{5}\right)_{i j}\left(\psi_{j \mu_{1} \mu_{2} \ldots \mu_{k}}^{v}\right)^{\dagger} \\
& -\left(\psi_{i v \mu_{1} \mu_{2} \ldots \mu_{k}}\right)\left(\gamma_{5}\right)_{i j}\left(\psi_{j \mu_{1} \mu_{2} \ldots \mu_{k}}^{\mu}\right)^{\dagger} \\
& +\left(F_{\mu \mu_{1} \mu_{2} \ldots \mu_{k}}\right)\left(G_{\mu_{1} \mu_{2} \ldots \mu_{k}}^{v}\right)^{\dagger}-\left(F_{v \mu_{1} \mu_{2} \ldots \mu_{k}}\right)\left(G_{\mu_{1} \mu_{2} \ldots \mu_{k}}^{\mu}\right)^{\dagger} \\
& \left.+\left(G_{\mu \mu_{1} \mu_{2} \ldots \mu_{k}}\right)\left(F_{\mu_{1} \mu_{2} \ldots \mu_{k}}^{v}\right)^{\dagger}-\left(G_{v \mu_{1} \mu_{2} \ldots \mu_{k}}\right)\left(F_{\mu_{1} \mu_{2} \ldots \mu_{k}}^{\mu}\right)^{\dagger}\right\}
\end{aligned}
$$

We record the following commutation relations for future use:

$$
\left[L_{\alpha \beta}, L_{\gamma \delta}\right]=\left\{\eta_{\alpha \delta} L_{\beta \gamma}+\eta_{\beta \gamma} L_{\alpha \delta}-\eta_{\alpha \gamma} L_{\beta \delta}-\eta_{\beta \delta} L_{\alpha \gamma}\right\}
$$

and

$$
\left[\tilde{L}_{\alpha \beta}, L_{\gamma \delta}\right]=\left\{\eta_{\alpha \delta} \tilde{L}_{\beta \gamma}+\eta_{\beta \gamma} \tilde{L}_{\alpha \delta}-\eta_{\alpha \gamma} \tilde{L}_{\beta \delta}-\eta_{\beta \delta} \tilde{L}_{\alpha \gamma}\right\}
$$

and

$$
\left[\tilde{L}_{\alpha \beta}, \tilde{L}_{\gamma \delta}\right]=\left\{\eta_{\alpha \delta} L_{\beta \gamma}+\eta_{\beta \gamma} L_{\alpha \delta}-\eta_{\alpha \gamma} L_{\beta \delta}-\eta_{\beta \delta} L_{\alpha \gamma}\right\} .
$$

Equation 181 is familiar. These equations contain two noncommuting copies of the commutation relations for the Lorentz group.

\section{A Simple Form for $\Delta_{2}$}

By using the above and utilizing the equations in Sect. 7 we can rewrite $\Delta_{2}$ in the form:

$$
\Delta_{2}=\Pi_{2}\left\{\nabla_{j}^{\dagger} \Pi_{\Lambda=0} \nabla_{i} P_{j i}^{+}+2(M-4) N_{1}^{\prime}+2 L_{\mu v} L^{\prime \mu \nu}\right\} \Pi_{2},
$$

where we define:

$$
L^{\prime \mu \nu}=\frac{1}{2} c_{i}\left(\gamma_{\mu \nu}\right)_{i j} c_{j}^{\dagger}=\frac{1}{2}\left(\gamma^{\nu \mu}\right)_{j i} c_{i} c_{j}^{\dagger} .
$$

This operator has been normalized so that it also satisfies the relations:

$$
\left[L_{\alpha \beta}^{\prime}, L_{\gamma \delta}^{\prime}\right]=\left\{\eta_{\alpha \delta} L_{\beta \gamma}^{\prime}+\eta_{\beta \gamma} L_{\alpha \delta}^{\prime}-\eta_{\alpha \gamma} L_{\beta \delta}^{\prime}-\eta_{\beta \delta} L_{\alpha \gamma}^{\prime}\right\}
$$

The operator $M$ can be written in several useful ways:

$$
M=D(f)+\frac{7}{2} N_{1}-\frac{3}{2} \hat{N}_{1}=4 N(F)+4 N(G)+2 N(\psi)+N(\partial),
$$

where $N(\partial)$ is the operator which counts the number of derivatives. The commutation relations of $M$ can be deduced from those of these operators. 


\section{The Operators $d_{r}$ are Zero for $r \geqq 3$}

It is easy to prove that the operators $d_{r}$ are zero for $r \geqq 3$ using the fact that all these operators contain the terms:

$$
d_{r}=\Pi_{r} \delta_{2} \delta_{0}^{\dagger} \frac{1}{\Delta_{0}} \ldots \Pi_{r}
$$

Now in the present case, this has the explicit form:

$$
\begin{gathered}
d_{r}=\Pi_{r}\left[c_{i} \nabla_{i}-\bar{c} \gamma_{\mu} c \varepsilon_{\mu}^{\dagger}\right], \\
{\left[\Lambda_{j}^{\dagger} c_{j}^{\dagger}+\varepsilon^{\mu \dagger} \partial_{\mu}^{\dagger}\right] \frac{1}{\Delta_{0}} \ldots \Pi_{r}=0,}
\end{gathered}
$$

where we use the (anti)commutation relations and the properties of the $\Pi_{r}$ operators established above:

$$
\begin{aligned}
\Pi_{r} \Lambda_{i}^{\dagger} & =0, \\
\Pi_{r} \varepsilon_{\mu}^{\dagger} & =0, \\
\left\{\nabla_{i}, \Lambda_{j}^{\dagger}\right\} & =0 .
\end{aligned}
$$

It follows that we will be finished when we have analyzed $E_{3}$, which we now proceed to do.

\section{A Set of Equations for the Space $E_{3}=E_{\infty}$}

Let us look for solutions $E_{3} C E_{2}$ for which:

$$
\Pi_{\Lambda=0} \nabla_{i} P_{j i}^{+} E_{3}=0 \text {. }
$$

By commuting this equation with the equation:

$$
c_{j}^{\dagger} \nabla_{j}^{\dagger} E_{3}=0,
$$

which is a consequence of the relations (166) and (167), we obtain:

$$
\left\{2(M-4) c_{i}^{\dagger}+L_{\mu v}\left(\gamma_{\mu v}\right)_{i j} c_{j}^{\dagger}\right\} E_{3}=0 \text {. }
$$

Now let us multiply this equation from the left by $c_{i}$ and by $c_{j}\left(\gamma_{5}\right)_{j i}$. This produces:

$$
\left\{2(M-4) N_{1}^{\prime}+2 L_{\mu v} L^{\prime \mu v}\right\} E_{3}=0
$$

and

$$
\left\{2(M-4) \tilde{N}_{1}^{\prime}-\varepsilon^{\alpha \beta \nu \delta} L_{\alpha \beta} L_{\gamma \delta}^{\prime}\right\} E_{3}=0 .
$$

The first of these already appears in 184. The second is used in deducing the equations below. Now let us multiply Eq. (193) from the left by $c_{i}\left(\gamma_{\alpha \beta}\right)_{i j}$ and then reduce the product of $\gamma$ matrices using (173). We get:

$$
\left\{4(M-4) L^{\alpha \beta}-2 L^{\alpha \beta} N_{1}^{\prime}-\varepsilon^{\alpha \beta \gamma \delta} L_{\gamma \delta} \tilde{N}_{1}^{\prime}+4 L_{\mu}^{\alpha} L^{\prime \mu \beta}-4 L_{\mu}^{\beta} L^{\prime \mu \alpha}\right\} E_{3}=0 .
$$

Now we can contract this equation from the left successively with $L_{\alpha \beta}$ and $L_{\beta \alpha}^{\prime}$ and using (194) and (195) we get two equations as follows:

$$
\begin{gathered}
\left\{2 L_{\mu \nu} L^{\nu \mu}-\mathscr{J} \varepsilon^{\alpha \beta \gamma \delta} L_{\alpha \beta} L_{\gamma \delta}-4(M-4)(M-2)\right\} E_{\infty}=0, \\
\left\{L_{\mu \nu}^{\prime} L^{\prime \nu \mu}-N_{1}^{\prime}\left(N_{1}^{\prime}+2\right)\right\} E_{\infty}=0 .
\end{gathered}
$$


These two and Eq. (194) can now be written in the form:

$$
\begin{gathered}
\left\{4(M-4)(M-2)-\hat{L}^{v \mu} \hat{L}_{\mu v}\right\} N_{1}^{\prime} E_{3}=0, \\
{[M-4]\left\{\hat{L}^{\prime \beta \alpha} \hat{L}_{\alpha \beta}^{\prime}-N_{1}^{\prime}\left(N_{1}^{\prime}+2\right)\right\} E_{3}=0,} \\
\left\{(M-4) N_{1}^{\prime}+\hat{L}_{\mu \nu} \hat{L}^{\prime \mu \nu}\right\} E_{\infty}=0,
\end{gathered}
$$

where the commutation relations of the $L$ operators and various properties like the following identity have been used:

$$
\begin{aligned}
\varepsilon^{\alpha \beta \gamma \delta} L_{\alpha \beta} L_{\gamma \delta} \tilde{N}_{1}^{\prime} \Pi_{2} & =\varepsilon^{\alpha \beta \gamma \delta} L_{\alpha \beta} \tilde{L}_{\gamma \delta} N_{1} \Pi_{2} \\
& =\mathscr{J} \varepsilon^{\alpha \beta \gamma \delta} L_{\alpha \beta} L_{\gamma \delta} N_{1} \Pi_{2} .
\end{aligned}
$$

Here we define

$$
\hat{L}_{\alpha \beta}=L_{\alpha \beta}-\frac{1}{2} \varepsilon_{\alpha \beta \gamma \delta} \tilde{L}^{\gamma \delta}=L_{\alpha \beta}-\frac{1}{2} \mathscr{J} \varepsilon_{\alpha \beta \gamma \delta} L^{\gamma \delta},
$$

and

$$
\hat{L}_{\alpha \beta}^{\prime}=L_{\alpha \beta}^{\prime}-\frac{1}{2} \varepsilon_{\alpha \beta \gamma \delta} \widetilde{L}^{\prime \gamma \delta} .
$$

Note that there are really only three operators (in each case) that are independent here:

$$
\hat{L}_{0 i}=-\frac{1}{2} \mathscr{J} \varepsilon_{i j k} \hat{L}_{j k}
$$

and

$$
\hat{L}_{0 i}^{\prime}=-\frac{1}{2} \mathscr{J} \varepsilon_{i j k} \hat{L}_{j k}^{\prime}
$$

In accord with standard notation we denote them by:

$$
\begin{aligned}
J_{i} & =\frac{1}{2} \mathscr{J} \hat{L}_{0 i}, \\
J_{i}^{\prime} & =\frac{1}{2} \mathscr{J} \hat{L}_{0 i}^{\prime} .
\end{aligned}
$$

Here of course $i, j, k$ take values in the set $1,2,3$. Now the commutation relation takes the form:

$$
\begin{aligned}
& {\left[J_{i}, J_{j}\right]=\mathscr{J} \varepsilon_{i j k} J_{k},} \\
& {\left[J_{i}^{\prime}, J_{j}^{\prime}\right]=\mathscr{J} \varepsilon_{i j k} J_{k}^{\prime},}
\end{aligned}
$$

and we also note that:

$$
\begin{gathered}
J_{i}^{\dagger}=J_{i}, \\
J_{i}^{\prime \dagger}=J_{i}^{\prime} .
\end{gathered}
$$

Let us now define a "total angular momentum operator" by:

$$
\hat{J}_{i}=J_{i}+J_{i}^{\prime}
$$

and observe that:

$$
J_{i} J_{i}^{\prime}=\frac{1}{2}\left\{\hat{J}_{i}^{2}-J_{i}^{2}-J_{i}^{\prime 2}\right\} .
$$

Our equations now take the form:

$$
N_{1}^{\prime}\left\{\left(\frac{M-4}{2}\right)\left(\frac{M-2}{2}\right)-J_{i} J_{i}\right\} E_{3}=0
$$


and

$$
(M-4)\left\{\frac{N_{1}^{\prime}}{2} \frac{\left(N_{1}^{\prime}+2\right)}{2}-J_{i}^{\prime} J_{i}^{\prime}\right\} E_{3}=0
$$

and

$$
\left\{\frac{\left[M-4+N_{1}^{\prime}\right]}{2} \frac{\left[M-2+N_{1}^{\prime}\right]}{2}-\hat{J}_{i} \hat{J}_{i}\right\} E_{3}=0 .
$$

Note that the $J_{i}$ and the $J_{i}^{\prime}$ form hermitian representations of the rotation group in three dimensions. This group is not the usual rotation group although it is related to it. The eigenvalues and eigenvectors of $J_{i} J_{i}$ are of course well known. Evidently we are generating here something related to the $\left(0, \frac{J}{2}\right)$ representations of the Lorentz group. However, once again, things are not quite so simple since there is no term in $L_{\mu \nu}$ that "rotates" the spinor according to the Lorentz group.

\section{Summary of the Equations for $E_{\infty}$}

Now we can collect together all the equations for $E_{\infty}$ in one place. First we have the equations that determine the subspace $E_{1}$ :

$$
\begin{gathered}
\Lambda_{i} E_{\infty}=0, \\
\partial_{\mu}^{\dagger} E_{\infty}=0, \\
N_{1} \varepsilon_{\mu}^{\dagger} \varepsilon_{\mu} E_{\infty}=0, \\
Q E_{\infty}=\left\{N_{1} N_{1}^{\prime}-\tilde{N}_{1} \tilde{N}_{1}^{\prime}\right\} E_{\infty}=0 .
\end{gathered}
$$

For the polynomials that have $N_{1}^{\prime} \geqq 1$ we also have the equation:

$$
T E_{\infty}=0 \text {. }
$$

For the polynomials that have $N_{1}^{\prime} \geqq 2$ we also have the equation:

$$
T^{\prime} E_{\infty}=0 \text {. }
$$

Next we list the equations that come from the conditions on $E_{3}$ :

$$
P_{i j}^{+} \Pi_{\Lambda=0} \nabla_{j} E_{\infty}=0 \text {. }
$$

For the polynomials that have $N_{1}^{\prime} \geqq 1$ we also have the equations:

$$
\begin{aligned}
& W E_{\infty}=0, \\
& W^{\prime} E_{\infty}=0, \\
& \hat{W} E_{\infty}=0,
\end{aligned}
$$

where we introduce the abbreviations:

$$
\begin{gathered}
W=2 L_{\mu \nu} L^{\nu \mu}-\mathscr{J} \varepsilon^{\alpha \beta \gamma \delta} L_{\alpha \beta} L_{\gamma \delta}-4(M-4)(M-2), \\
W^{\prime}=\left[L_{\mu \nu}^{\prime} L^{\prime v \mu}-N_{1}^{\prime}\left(N_{1}^{\prime}+2\right)\right](M-4), \\
\tilde{W}=(M-4) N_{1}^{\prime}+L_{\mu \nu} L^{\prime \mu \nu} .
\end{gathered}
$$




\section{Solutions of the Equations for $E_{3}$}

\subsection{Superfields and the $\mathscr{G}=0$ Sector of $E_{\infty}$}

At this point we will consider the construction of some solutions to this set of equations. The easiest place to start is with matters that are already well known the construction of the ghost degree zero polynomials in $H$. The best general known way of doing this is to use superspace methods. This is well explained in many references [7]. Here we only review the changes needed in our own context. There are three kinds of superfields:

1. Complex chiral superfields $S$, which are composed of complex component fields and complex chiral spinors and satisfy the relation:

$$
\overline{\mathscr{D}}_{\alpha} S=0 \text {, }
$$

where $\overline{\mathscr{D}}_{\alpha}$ is the antichiral superspace covariant derivative in the two component notation.

2. Complex antichiral superfields $\bar{S}$, which are composed of complex component fields and complex chiral spinors and satisfy the relation:

$$
\mathscr{D}_{\alpha} \bar{S}=0,
$$

where $\mathscr{D}_{\alpha}$ is the chiral superspace covariant derivative in the two component notation. If $S$ is a chiral superfield then its complex conjugate $\bar{S}$ is an antichiral superfield.

3. Real general superfields $V$, which are composed of real bosonic fields and chiral spinors in pairs to make Majorana spinors. They satisfy the relation:

$$
V=\bar{V} \text {. }
$$

In the present paper we have started with the complex chiral superfield $S$ of the form:

$$
S=\exp \left(-i \theta \not \bar{\phi} \overline{)}\left[(A+i B)+2\left(\theta_{\alpha} \psi^{\alpha}\right)-\varepsilon_{\alpha \beta} \theta^{\alpha} \theta^{\beta}(F+i G)\right]\right.
$$

and we are permitted to make other superfields from it according to the following rules:

1. The product of two superfields is a superfield, and multiplication of superfields is associative, so that the product of any number of superfields is a superfield.

2. The chiral derivative $\mathscr{D}_{\alpha}$ of a superfield is a superfield.

3. The antichiral derivative $\overline{\mathscr{D}}_{\alpha}$ of a superfield is a superfield.

4. The spacetime derivative of a superfield is a superfield.

The following criteria determine the nature of the superfield so formed:

1. The product of three or more chiral derivatives is zero, because of their anticommutativity.

2. The product of three or more antichiral derivatives is zero, because of their anticommutativity.

3. Superfields formed by taking a product or a chiral derivative or some combination of both are not in general either chiral or antichiral or real. To form one of the three irreducible kinds of superfields one can do the following (sometimes the result is zero): 
(a) A chiral field can be formed from this object by acting with the operator $\overline{\mathscr{D} \mathscr{D}}$.

(b) An antichiral field can be formed from this object by acting with the operator $\mathscr{D} \mathscr{D}$.

(c) A real superfield can be formed by taking the real or imaginary part of this object.

4. Superfields may have uncontracted Lorentz or spinor indices. This does not affect their status as superfields. Such indices may arise in the present case from uncontracted chiral or antichiral or spacetime derivatives.

Once one has constructed superfields, one can then construct superspace invariants from them by doing superspace integrals of them. Chiral superfields must be integrated over chiral superspace and real superfields must be integrated over all of superspace. The chiral integration of a chiral superfield is a complex quantity of course. It is just the " $F+i G$ " part of the superfield. Normally one picks out the real part of this complex quantity and ignores the other one. An alternative is to take the imaginary part $G$. This will also be a superspace invariant. It will have the opposite parity to the $F$ part. This is important for our purposes. Real superfields must be integrated over all of superspace - both chiral and antichiral parts. This picks out the $D$ part of the real superfield. No " $G$ " type invariant exists in this case.

Now let us make contact with the formalism of this paper. The invariants in $H$ with $\mathscr{G}=0$ give rise to polynomials in $E_{\infty}$ with $\mathscr{G}=0$ and $\mathscr{F}=N(\varepsilon)=4$ as follows: Denote the $F, G$ or $D$ term of any superfield by $F^{\prime}, G^{\prime}$ or $D^{\prime}$ respectively. Write these (illustrated for $F^{\prime}$ only) in the form:

$$
F^{\prime}=\sum_{k=q_{1}}^{k=q_{2}} F_{q}^{\prime}
$$

where the summands are eigenstates of the grading operator $N$ :

$$
N F_{q}^{\prime}=q F_{q}^{\prime} \text {. }
$$

Then $F_{q_{1}}^{\prime} T_{\varepsilon}^{4}$ will always be in $E_{\infty}$. For simple cases it turns out that the terms of the form $F^{\prime}$ and $G^{\prime}$ give rise to polynomials in $E_{\infty}$ with $T=0$ and the terms of the form $D^{\prime}$ give rise to polynomials in $E_{\infty}$ with $T>0$. I conjecture that this is a general rule. Since the $F^{\prime}, G^{\prime}$, and $D^{\prime}$ can have uncontracted Lorentz or spinor indices, so can the polynomials found in $E_{\infty}$.

\subsection{Construction of Multiplets}

The theory of construction of superspace invariants is simplest in superspace and was discussed above. However to make contact with our previous formalism, it is easiest to revert to the old style of construction of invariants using the "multiplication of multiplets" method. This is equivalent to the superspace method. As is well known, one can multiply two chiral supersymmetry multiplets to get a third. In our notation this takes the form:

$$
\begin{aligned}
& A^{\prime \prime}=A A^{\prime}-B B^{\prime}, \\
& B^{\prime \prime}=A B^{\prime}+B A^{\prime}, \\
& \psi^{\prime \prime}=\left(A-B \gamma_{5}\right) \psi^{\prime}+\left(A^{\prime}-B^{\prime} \gamma_{5}\right) \psi, \\
& F^{\prime \prime}=F A^{\prime}+F^{\prime} A+G B^{\prime}+G^{\prime} B-\bar{\psi} \psi^{\prime}, \\
& G^{\prime \prime}=G A^{\prime}+G^{\prime} A-F B^{\prime}-F^{\prime} B+\bar{\psi} \gamma_{5} \psi^{\prime},
\end{aligned}
$$


and it satisfies the equations:

$$
\begin{gathered}
\delta A^{\prime \prime}=\bar{c} \psi^{\prime \prime}+\varepsilon^{\mu} \partial_{\mu} A^{\prime \prime}, \\
\delta B^{\prime \prime}=\bar{c} \gamma_{5} \psi^{\prime \prime}+\varepsilon^{\mu} \partial_{\mu} B^{\prime \prime}, \\
\left.\delta \psi^{\prime \prime}=\not A^{\prime \prime}+B^{\prime \prime} \gamma_{5}\right] c+\left[F^{\prime \prime}+G^{\prime \prime} \gamma_{5}\right] c+\varepsilon^{\mu} \partial_{\mu} \psi^{\prime \prime}, \\
\delta F^{\prime \prime}=\bar{c} \not \psi^{\prime \prime}+\varepsilon^{\mu} \partial_{\mu} F^{\prime \prime} \\
\delta G^{\prime \prime}=\bar{c} \gamma_{5} \not \psi^{\prime \prime}+\varepsilon^{\mu} \partial_{\mu} G^{\prime \prime}
\end{gathered}
$$

whenever the corresponding equations are satisfied for the superfields $S$ and $S^{\prime}$. This is simply what one obtains by multiplying two chiral superfields and then writing down the components of the resulting chiral superfield.

\subsection{Some Polynomials with $\mathscr{G} \geqq 1$ : Solutions with no Spacetime Derivatives}

Let us collect together and list all the equations that must be satisfied by the polynomials $P$ in $E_{3}=E_{\infty}$ that have $\mathscr{G}=1$ :

$$
\Lambda_{i} P=0, \quad \partial_{\mu}^{\dagger} P=0, \quad T P=0, \quad N_{1} \varepsilon_{\mu}^{\dagger} \varepsilon_{\mu} P=0, \quad\left[1-\mathscr{J} \mathscr{J}^{\prime}\right] P=0 .
$$

These express the requirement that $P$ is in the $\mathscr{G}=1$ part of the subspace $E_{1}=E_{2}$. The simplest solutions are those which contain no spacetime derivatives. For these the equations of the subspace $E_{2}$ reduce simply to:

$$
\Pi_{\Lambda=0} \nabla_{i} P_{j i}^{+} P=0
$$

and

$$
(M-4) P=0
$$

since the absence of derivatives makes the equation:

$$
L_{\mu \nu} P=0
$$

automatic.

The solutions of these equations are easy to find. First one considers all the possible elements of $H$ with $\mathscr{G}=0$. These can be constructed using superfields or multiplet combination as outlined above. We want only those for which the lowest $N$ part of the superfield is free of spacetime derivatives. Since we want $T=0$, we take only the $F^{\prime}$ and $G^{\prime}$ parts of chiral multiplets. Then the equation $M=4 N(F)$ $+4 N(G)+2 N(\psi)+N(\partial)=4$ means that the required lowest $N$ part of the superfield $F^{\prime}, G^{\prime}$ or $D^{\prime}$ components must be a sum of terms linear in $F$ and $G$ and bilinear in $\psi$. So we want terms that are the $F^{\prime}$ and $G^{\prime}$ components of composite chiral multiplets, and are linear in $F$ and $G$ or bilinear in $\psi$.

Then we have to satisfy Eq. (218) above. This is easy to do with the combinations below. The number of $A$ or $B$ fields is unconstrained by the $M-4=0$ equation. The first few solutions in the infinite series that is so generated are:

$$
\begin{gathered}
{\left[F c_{i}-G\left(\gamma_{5}\right)_{i j} c_{j}\right] T_{\varepsilon}^{4},} \\
\left\{\left[A F+B G-\frac{1}{2} \bar{\psi} \psi\right] c_{i}-\left[A G-B F+\frac{1}{2} \bar{\psi} \gamma_{5} \psi\right]\left(\gamma_{5}\right)_{i j} c_{j}\right\} T_{\varepsilon}^{4}, \\
\left\{\left\{\left(A^{2}-B^{2}\right) F+2 A B G-\bar{\psi}\left(A-B \gamma_{5}\right) \psi\right\} c_{i}\right. \\
\left.-\left\{\left(A^{2}-B^{2}\right) G-2 A B F+\bar{\psi}\left(B+A \gamma_{5}\right) \psi\right\}\left(\gamma_{5}\right)_{i j} c_{j}\right\} T_{\varepsilon}^{4}
\end{gathered}
$$


Now these correspond simply with the following objects in $H$ :

$$
\begin{gathered}
\int d^{4} x\left[F c_{i}-G\left(\gamma_{5}\right)_{i j} c_{j}\right] \in H, \\
\left.\int d^{4} x\left\{\left[A F+B G-\frac{1}{2} \bar{\psi} \psi\right] c_{i}-\left[A G-B F+\frac{1}{2} \bar{\psi} \gamma_{5} \psi\right]\left(\gamma_{5}\right)_{i j} c_{j}\right]\right\}, \\
\int d^{4} x\left\{\left\{\left(A^{2}-B^{2}\right) F+2 A B G-\bar{\psi}\left(A-B \gamma_{5}\right) \psi\right\} c_{i}\right. \\
\left.-\left\{\left(A^{2}-B^{2}\right) G-2 A B F+\bar{\psi}\left(B+A \gamma_{5}\right) \psi\right\}\left(\gamma_{5}\right)_{i j} c_{j}\right\}
\end{gathered}
$$

It is easy to see that the first of these objects is indeed in the cohomology space $H$, but this is not so evident for the second or third. This can be verified simply by acting on these polynomials with the operator $\delta^{\dagger}$ as defined from Eq. (12) after it have been projected with the operators $\Pi_{\partial^{\dagger}=0}$ and $\Pi_{c=0}$ corresponding to the spectral sequence generated by the grading operator $N=c_{i} c_{i}^{\dagger}$ as is done in [2] to prove the isomorphism of the cohomology of $\delta$ in the spaces $P$ and $L$. This shows in a straightforward way that these objects are indeed in $H$ and provides some independent confirmation of the long derivation above.

There is clearly an infinite series of such objects all of which are in $E_{3}$ and which correspond simply with objects in $H$. They all have the property that they satisfy the equation:

$$
L_{\mu \nu} P=0
$$

trivially by virtue of the fact that they contain no derivatives. We note that these solutions generalize easily to higher values of the ghost charge. One simply takes combinations like:

$$
F \bar{c} \gamma_{\mu \nu} c-G \bar{c} \gamma_{\mu \nu} \gamma_{5} c
$$

and a similar discussion can be given. It is easy to verify that the equation:

$$
T^{\prime} \bar{c} \gamma_{\mu \nu} c
$$

is satisfied. There appear to be solutions of this kind for all values of the ghost charge up to $\infty$.

\section{Part IV. Conclusion}

As was stated in the Introduction, the hope that motivates the present paper is that there may exist supersymmetry anomalies that have not yet been uncovered, and that if these exist, they may have some use. The result of the analysis here is quite encouraging. It indicates that the cohomology space for the simplest supersymmetric BRS operator is far more complicated than the cohomology spaces of Yang-Mills theory and gravitation. In particular there are an infinite number of polynomials in the $\mathscr{G}=1$ sector of the cohomology space.

This is a bit of a surprise because it has generally been assumed that making a theory supersymmetric does not materially change the cohomology structure of the theory. It has been expected that the ghost charge one polynomials in supersymmetric theories would be simply the ghost charge one polynomials put into supersymmetric form somehow.

Hence this result casts some doubt on the conjecture that there are no anomalies beyond these supersymmetric versions of the known anomalies.

Since the cohomology space is not empty, the natural reaction to this result would be to calculate the Feynman diagrams that correspond with the objects in 
the cohomology space to see if there are anomalies in the theory. The cohomology space of ghost charge one tells one where to look for anomalies. The present result indicates that there are an infinite number of places to look (assuming that one can construct appropriate operators of ghost charge zero) and it describes them.

More accurately, the result would indicate where to look except that there is a supersymmetric regularization for the simple Wess Zumino multiplet [8]. However if this multiplet is coupled to a theory like supersymmetric Yang-Mills theory, then we might expect the objects found here in the cohomology space to be anomalies. In the case of supersymmetric Yang-Mills theory, no-one has ever succeeded in constructing a manifestly supersymmetric and gauge invariant regularization procedure.

In [3] it is shown that the present analysis does in fact generalize to super YangMills quite easily, and the consequences of that are discussed there.

This in turn raises the question of what happens in a theory such as the superstring. Is there a chance that the "compactification" of the superstring or the breaking of supersymmetry invariance is driven by supersymmetry anomalies in some way? Only an evaluation of the BRS cohomology of these theories can provide an answer.

\section{Part V. Appendices}

\section{A. Summary of Basic Results and Notation}

The techniques in this paper were introduced in [2]. This appendix is a reminder of the results and notation established there, and it also summarizes the new notation used in this paper.

$L$ is the linear vector space of integrated polynomials in the Wess Zumino fields $A, B, \psi, F, G$, their derivatives $\partial_{\mu}$ of all orders and the spacetime independent commuting Majorana spinor supersymmetry ghost field $c . P$ is the space of unintegrated polynomials in the Wess Zumino fields $A, B, \psi, F, G$, their derivatives $\partial_{\mu}$ of all orders, the spacetime independent commuting Majorana spinor $c$ and the spacetime independent anticommuting translation ghost field $\varepsilon_{\mu}$. The combination $\varepsilon_{0} \varepsilon_{1} \varepsilon_{2} \varepsilon_{3}$ is denoted $T_{\varepsilon}^{4}$.

The variables that we use are of the form $A_{\mu_{1} \mu_{2} \ldots \mu_{k}}$ which is an abbreviation for $\partial_{\mu_{1}} \partial_{\mu_{2}} \ldots \partial_{\mu_{k}} A$. We promote these to independent variables in Fock space, with commutation relations:

$$
\left[A_{\mu_{1} \mu_{2} \ldots \mu_{k}}^{\dagger}, A_{v_{1} v_{2} \ldots v_{k}}\right]=\left\{\delta_{\mu_{1} v_{1}} \delta_{\mu_{2} v_{2}} \ldots \delta_{\mu_{k} v_{k}}+\delta_{\mu_{1} v_{2}} \delta_{\mu_{2} v_{1}} \ldots \delta_{\mu_{k} v_{k}}+\ldots\right\},
$$

where the sum is performed over all $k$ ! permutations of the variables. Note that the weight is as shown. There are $k$ ! terms here and no factor of $1 / k$ ! in front. (Anti)commutation relations for the other variables are all defined the same way.

The nilpotent operator $\delta$ is defined by 11 when acting on $L$ and by 12 when acting on $P$. The cohomology of $\delta$ in $L$ is denoted by $H_{L}$, and the cohomology of $\delta$ in $P$ is denoted by $H_{P}$. These two are isomorphic as is shown in [2].

The advantage of using the positive definite inner product in Fock space is that the following isomorphism holds:

$$
H_{P}=\operatorname{Ker} \Delta,
$$

where $\Delta$ is defined to be the non-negative operator:

$$
\Delta=\left[\delta+\delta^{\dagger}\right]^{2} .
$$


The spectral sequence is generated by a grading operator $N$. It consists of a nested series of spaces $E_{r}$ which are subspaces of $P$. A sequence of nilpotent operators $d_{r}$ acting in the subspaces $E_{r}$ can be constructed. Each space $E_{r+1}$ is the cohomology space of the operator $d_{r}$ acting in the space $E_{r}$. It can be computed using the formula $E_{r+1}=E_{r} \cap \operatorname{Ker} \Delta_{r}$, where $\Delta_{r}=\left(d_{r}+d_{r}^{\dagger}\right)^{2}$. The orthogonal projection operator $\Pi_{r}$ onto the space $E_{r}$ is needed to define the operator $d_{r}$. The spectral sequence is said to converge to $E_{q}$ for some positive integer $q$ when $d_{r}=0$ for $r \geqq q$. Then we denote the resulting space $E_{\infty}=E_{q}$. The important theorem is that this space $E_{\infty}$ is isomorphic to $H_{P}$ which in turn is isomorphic to $H_{L}$.

We defined $\Lambda_{i}$ and $\nabla_{i}$ to be pieces of $\delta$ in Eqs. (14) and (15). The dimension operator $D$ and the ghost $\mathscr{G}$ and form $\mathscr{F}$ degree operators are defined in Sect. 8.

The operator $N_{1}$ is the counting operator. It counts the number of fields in a polynomial. $\widetilde{N}_{1}$ is a "twisted" counting operator defined in 76. Other "twisted" operators are frequently defined and used in the paper. The twisted operator is always closely related to the untwisted one with the same symbol. $T$ is defined as an abbreviation of $N_{1}^{2}+\tilde{N}_{1}^{2}$. The notation $N(A)$ is used for the counting operator for the field $A$ alone. The operator $N_{1}^{\prime}$ is the counting operator for the $c$ field. It counts the number of $c$ fields in a polynomial. $\widetilde{N}_{1}^{\prime}$ is a twisted counting operator defined in 104. $T^{\prime}$ is defined as an abbreviation of $N_{1}^{\prime 2}+\widetilde{N}_{1}^{\prime 2}$.

The operator $Q$ is defined as an abbreviation of $N_{1} N_{1}^{\prime}-\tilde{N}_{1} \tilde{N}_{1}^{\prime}$. The operator $\mathscr{J}$ acts like $i=\sqrt{-1}$. It is defined by $\mathscr{J}=\tilde{N}_{1} / N_{1}$. The operator $\mathscr{J}^{\prime}$ is defined by $\mathscr{J}^{\prime}=\tilde{N}_{1}^{\prime} / N_{1}^{\prime}$. It acts like $-i=-\sqrt{-1}$. The notation $v_{\mu}$ stands for the important combination $\bar{c} \gamma_{\mu} c$. The frequently used notations $\Pi_{X=0}$ and $\Pi_{X>0}$ are defined in Sect. 8. The operators $M, \tilde{M}, L_{\mu \nu}$, and $\tilde{L}_{\mu \nu}$ are all defined in Sect. 14. The operator $L_{\mu \nu}^{\prime}$ is defined in Sect. 15. The operators $\hat{L}_{\mu \nu}$ and $\hat{L}_{\mu \nu}^{\prime}$ and the angular momentum operators $J_{i}, J_{i}^{\prime}$, and $\hat{J}_{i}$ are all defined in Sect. 17 . The operators $W, W^{\prime}$, and $\tilde{W}$ are defined in Sect. 18.

\section{B. $\gamma$ Matrix Conventions and Formulae}

The $\gamma$ matrices in this paper are in a real (Majorana) representation and the Lorentz metric is defined by the relation:

$$
x_{\mu} x^{\mu}=\eta_{\mu v} x^{\mu} x^{\nu}=-x_{0}^{2}+x_{1}^{2}+x_{2}^{2}+x_{3}^{2} .
$$

The $\gamma$ matrices satisfy the following relation:

$$
\left\{\gamma_{\mu}, \gamma_{v}\right\}=2 \eta_{\mu \nu} \text {. }
$$

We also use the "Kronecker delta" which is defined by:

$$
x_{\mu} x_{\mu}=\delta_{\mu v} x_{\mu} x_{v}=+x_{0}^{2}+x_{1}^{2}+x_{2}^{2}+x_{3}^{2} .
$$

The matrix $\gamma_{5}$ is defined by:

$$
\gamma_{5}=\gamma_{0} \gamma_{1} \gamma_{2} \gamma_{3}
$$

and the following identities hold:

$$
\begin{gathered}
\gamma_{\mu}=\gamma_{\mu}^{*}, \\
\gamma_{0} \gamma_{\mu} \gamma_{0}=\gamma_{\mu}^{T}=\gamma^{\mu}, \\
\gamma_{5}=\gamma_{5}^{*}=-\gamma_{5}^{T},
\end{gathered}
$$


where * denotes the complex conjugate and $T$ denotes the transpose of a matrix. For convenience we record the following relations which are easily derived:

$$
\begin{gathered}
\gamma_{5}^{2}=-1, \\
\left(\gamma_{0}\right)^{T}=-\gamma_{0}, \\
\left(\gamma_{0} \gamma_{5}\right)^{T}=-\gamma_{0} \gamma_{5}, \\
\left(\gamma_{0} \gamma_{\mu}\right)^{T}=+\gamma_{0} \gamma_{\mu}, \\
\left(\gamma_{0} \gamma_{\mu} \gamma_{5}\right)^{T}=-\gamma_{0} \gamma_{\mu} \gamma_{5}, \\
\left(\gamma_{0} \gamma_{\mu \nu}\right)^{T}=+\gamma_{0} \gamma_{\mu \nu},
\end{gathered}
$$

where we define:

$$
\gamma_{\mu \nu}=\frac{1}{2}\left[\gamma_{\mu}, \gamma_{\nu}\right]
$$

The Fierz identity for commuting spinors takes the form:

$$
\begin{aligned}
\bar{A} D \bar{C} B= & \frac{1}{4}\left[\bar{A} B \bar{C} D-\bar{A} \gamma_{5} B \bar{C} \gamma_{5} D+\bar{A} \gamma_{\mu} B \bar{C} \gamma^{\mu} D\right. \\
& \left.+\bar{A} \gamma_{\mu} \gamma_{5} B \bar{C} \gamma^{\mu} \gamma_{5} D-\frac{1}{2} \bar{A} \gamma_{\mu \nu} B \bar{C} \gamma^{\mu \nu} D\right],
\end{aligned}
$$

where we use the usual notation:

$$
\bar{A} B \bar{C} D=A_{i}^{*} B_{j}\left(\gamma_{0}\right)_{i j} C_{p}^{*} D_{q}\left(\gamma_{0}\right)_{p q},
$$

and of course in this representation for Majorana spinors we have:

$$
A=A^{*} \text {. }
$$

The following identities are easily proved:

$$
\begin{gathered}
\gamma_{\mu \nu} \gamma_{5}=\frac{1}{2} \varepsilon_{\mu \nu \lambda \sigma} \gamma^{\lambda \sigma}, \\
\gamma_{\alpha \beta} \gamma_{\gamma \delta}=\eta_{\alpha \delta} \eta_{\beta \gamma}-\eta_{\alpha \gamma} \eta_{\beta \delta}+\varepsilon_{\alpha \beta \gamma \delta} \gamma_{5}+\gamma_{\alpha \delta} \eta_{\beta \gamma}+\gamma_{\beta \gamma} \eta_{\alpha \delta}-\gamma_{\alpha \gamma} \eta_{\beta \delta}-\gamma_{\beta \delta} \eta_{\alpha \gamma}, \\
\gamma_{\alpha} \gamma_{\beta \gamma}=\eta_{\alpha \beta} \gamma_{\gamma}-\eta_{\alpha \gamma} \gamma_{\beta}-\varepsilon_{\alpha \beta \gamma \sigma} \gamma^{\sigma} \gamma_{5}, \\
\gamma_{\mu \nu} \gamma^{\mu \nu}=-12, \\
\gamma_{\mu} \gamma^{\mu}=4, \\
\gamma_{\mu \sigma} \gamma_{\nu}{ }^{\sigma}=-\left[3 \eta_{\mu \nu}+2 \gamma_{\mu \nu}\right],
\end{gathered}
$$

where we choose the convention $\varepsilon_{0123}=-\varepsilon^{0123}=+1$ and $\varepsilon_{\mu \nu \lambda \sigma}$ is the totally antisymmetric tensor in four dimensions. All of the above is independent of the specific choice made for the $\gamma$ matrices so long as they are real. We use the following specific (block matrix) four dimensional representation for the $\gamma$ matrices:

$$
\begin{aligned}
& \gamma_{0}=\left(\begin{array}{cc}
0 & -\sigma_{1} \\
\sigma_{1} & 0
\end{array}\right), \\
& \gamma_{1}=\left(\begin{array}{rr}
-1 & 0 \\
0 & +1
\end{array}\right), \\
& \gamma_{2}=\left(\begin{array}{cc}
0 & i \sigma_{2} \\
-i \sigma_{2} & 0
\end{array}\right),
\end{aligned}
$$




$$
\begin{aligned}
& \gamma_{3}=\left(\begin{array}{rr}
0 & -1 \\
-1 & 0
\end{array}\right), \\
& \gamma_{5}=\left(\begin{array}{cc}
0 & -\sigma_{3} \\
\sigma_{3} & 0
\end{array}\right),
\end{aligned}
$$

where the $2 \times 2$ sigma matrices are defined as usual:

$$
\begin{aligned}
& \sigma_{1}=\left(\begin{array}{ll}
0 & 1 \\
1 & 0
\end{array}\right), \\
& \sigma_{2}=\left(\begin{array}{cc}
0 & -i \\
i & 0
\end{array}\right), \\
& \sigma_{3}=\left(\begin{array}{rr}
1 & 0 \\
0 & -1
\end{array}\right)
\end{aligned}
$$

and the two dimensional unit matrix is of course:

$$
1=\left(\begin{array}{ll}
1 & 0 \\
0 & 1
\end{array}\right)
$$

We note the useful identity:

$$
\bar{c} \gamma_{\mu} c \bar{c} \gamma^{\mu}=0
$$

which follows from the Fierz identity for commuting spinors $c$.

Acknowledgements. I thank M. Dixon, M. Duff, P. Hawkes, R. Newberry, and K. Stelle for support and encouragement while this work was being done. I also thank O. Piguet, P. West, R. Stora, and J.C. Taylor for useful discussions and correspondence.

\section{References}

1. Becchi, C., Rouet, A., Stora, R.: Renormalization of gauge theories. Ann. Phys. 98, 127 (1975). See more complete references in [2]

2. Dixon, J.A.: Calculation of BRS cohomology using spectral sequences. Commun. Math. Phys. 139, 495-526 (1991)

3. Ibid.: Class. Quant. Grav. 7, 1511 (1990)

4. Fadeev, L.: Operator anomaly for the Gauss law. Phys. Lett. 145B, 81 (1984)

5. Green, M., Schwarz, J., Witten, E.: Superstring theory. Cambridge: Cambridge University Press 1987

6. Ferrara, S.: Supersymmetry. Amsterdam, Hong Kong: North-Holland-World Scientific 1987 contains many useful reprints. There are also a number of good texts

7. Gates, J., Grisaru, M. Roček, M., Siegel, W.: Superspace. London, Amsterdam: Benjamin 1983

8. See for example: Iliopoulos, J., Zumino, B.: Broken supergauge symmetry and renormalization. Nucl. Phys. B 76, 310 (1974)

9. Piguet, O., Schweda, M., Sibold, K.: Nucl. Phys. B 174, 183 (1980)

10. Piguet, O., Sibold, K.: Renormalized supersymmetry, Progress in Physics Series, Vol. 12. Cambridge, MA: Birkhauser 1986

11. Wess, J., Zumino, B.: Supergauge transformations in four dimensions. Nucl. Phys. B 70, 39 (1974)

Communicated by A. Jaffe 
